\title{
Biomechanical Stability of Dental Implants in Augmented Maxillary Sites: Results of a Randomized Clinical Study with Four Different Biomaterials and PRF and a Biological View on Guided Bone Regeneration
}

\author{
Troedhan Angelo, ${ }^{1}$ Wainwright Marcel, $^{2}$ Kurrek Andreas, ${ }^{3}$ and Schlichting Izabela ${ }^{4}$ \\ ${ }^{1}$ Institute for Maxillofacial Surgery and Dentistry, General Hospital of Vienna "Hietzing", Wolkersbergenstraße 1, \\ Pavillon 3a, 1130 Vienna, Austria \\ ${ }^{2}$ Implantology Clinic Kaiserswerth, Kaiserswerther Markt 25, 40489 Dusseldorf, Germany \\ ${ }^{3}$ Implantology Clinic Oberkassel, Dominikanerstrasse 10, 40545 Dusseldorf, Germany \\ ${ }^{4}$ Center for Facial Esthetics Vienna, Brauhausgasse 12, 1050 Vienna, Austria \\ Correspondence should be addressed to Troedhan Angelo; troed@aon.at
}

Received 1 January 2015; Revised 22 February 2015; Accepted 27 February 2015

Academic Editor: Devorah Schwartz-Arad

Copyright (c) 2015 Troedhan Angelo et al. This is an open access article distributed under the Creative Commons Attribution License, which permits unrestricted use, distribution, and reproduction in any medium, provided the original work is properly cited.

\begin{abstract}
Introduction. Bone regenerates mainly by periosteal and endosteal humoral and cellular activity, which is given only little concern in surgical techniques and choice of bone grafts for guided bone regeneration. This study investigates on a clinical level the biomechanical stability of augmented sites in maxillary bone when a new class of moldable, self-hardening calcium-phosphate biomaterials (SHB) is used with and without the addition of Platelet Rich Fibrin (aPRF) in the Piezotome-enhanced subperiosteal tunnel-technique (PeSPTT). Material and Methods. 82 patients with horizontal atrophy of anterior maxillary crest were treated with PeSPTT and randomly assigned biphasic (60\% HA/40\% bTCP) or monophasic (100\% bTCP) SHB without or with addition of aPRF. 109 implants were inserted into the augmented sites after 8.3 months and the insertion-torque-value (ITV) measured as clinical expression of the (bio)mechanical stability of the augmented bone and compared to ITVs of a prior study in sinus lifting. Results. Significant better results of (bio)mechanical stability almost by two-fold, expressed by higher ITVs compared to native bone, were achieved with the used biomaterials and more constant results with the addition of aPRF. Conclusion. The use of SHB alone or combined with aPRF seems to be favourable to achieve a superior (bio)mechanical stable restored alveolar bone.
\end{abstract}

\section{Introduction}

Physiology of bone and bone-healing after mechanical trauma (natural and iatrogenic macroscopic and/or microscopic fractures) is well known in general medicine for long [1] and biologically defined by the core function of peri- and endosteum [2,3] based on its histologic composition [4].

A "de novo" bone formation under the elevated Schneiderian membrane in sinus-lift-procedures [5] even in absence of auto-/hetero-/xenogeneic or synthetic biomaterials [6] was proven experimentally and clinically since the Schneiderian membrane is composed of mainly periosteum, lining the bony walls of the maxillary sinus [7] (Figure 1).

Obviously the known fact of vital peri- and endosteum to be the core carrier of bone healing and regeneration was long neglected in oral surgery and implantology [8], leading to numerous studies focusing on hypothetical osteoinductivity and/or osteoconductivity of auto-, heterologous, and xenogeneic bone grafts versus synthetic biomaterials [915]. Only recently it was investigated if the applied surgical technique, for example, sinus lifting, preserved an intact and vital elevated periosteum or merely led to a dissection of 


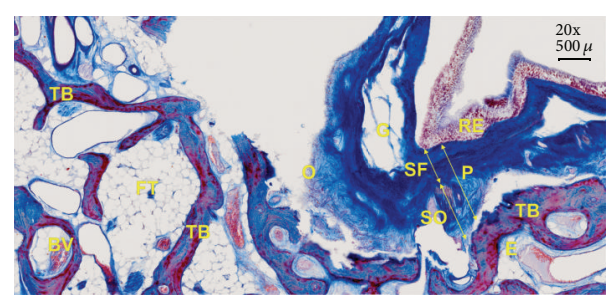

FIGURE 1: Section through a human maxillary sinus specimen after detachment of the Schneiderian membrane with the tHUCSLINTRALIFT-method in a fresh human cadaver head. RE: respiratory epithelium pointing inside the maxillary sinus, $\mathrm{P}$ : periosteum (total), SF: subsection of the periosteum (P): fibrous layer ("stratum fibrosum"), SO: subsection of the periosteum (P): osteogenic layer ("stratum osteogenicum"), O: osteoblasts (red dots), TB: trabecular bone (reddish), E: endosteum (blue layer covering all TB), G: gland, BV: blood vessel, and FT: fat tissue. Specimen at 20x magnification was prepared by immersion fixation in 5\%-neutral-formaldehydesolution, dehydrated with alcohol, embedded in Paraplast, and cut with a microtome to slices of 5-6 microns. Azan-staining was performed in order to visualize the osteoblasts within the periosteal layer (reddish color), the collagenous fibres of the periosteum and connective tissues of the sinus-membrane, and the connecting Sharpey fibres (dark blue color).

the periosteum from the respiratory epithelium [16], which leads to failure in guided-bone-regeneration (GBR) procedures. Contrary, a proven clean periosteal detachment [7] provides higher and constant success-rates in sinus lifting [17] even in the less remodeling-active posterior maxilla [18].

Both from biological and physiological standpoint, sinus lift procedures, regardless if performed with lateral or transcrestal approach, technically have to be considered as "subperiosteal tunnel" or "pocket" techniques, creating a bonebased and enclosed subperiosteal scaffold for guided bone regeneration (GBR) without the need to raise a full thickness mucoperiosteal flap especially in transcrestal procedures [17].

The subperiosteal tunnel technique (SPTT) for reconstruction of atrophic alveolar crests in the maxilla and mandible was first described in 1982 [19] and later evaluated with increasing patient numbers using nonporous hydroxyapatite (HA) ceramics [20] but suffered from displacement of the loose granules in the healing period [21]. Improvements of the technique for more predictable results were presented 1991, using nonporous HA, locked-up in a resorbable Vicryltube [22] or microporous HA with mechanical stabilization of the subperiosteal tunnel by a surgical splint [23]. These techniques enhanced the results achieved with SPTT regarding vertical height gain of the atrophic mandible by better immobilization of the synthetic bone graft and allowed a better stability of removable overdentures. Different biomaterials and addition of recombinant human platelet-derived growth factor $\mathrm{BB}$ (rhPDGF-BB) were investigated regarding bone regeneration with SPTT [24], matching the results achieved with the same biomaterials and rhPDGF-BB in sinus lift procedures [25] as well as injectable hyaluronic acidbased hydrogels with nanohydroxyapatite [26]. Nevertheless, the known absolute demand for a proper immobilization of a metallic implant into bone beyond a biological threshold to achieve full osseointegration of orthopedic $[27,28]$ and dental implants [29] was long neglected for guided-bone-regeneration-surgeries (GBR) such as SPTT and has to be applied also to particulate bone grafts used for GBR in oral surgery: they follow exactly the same pattern of osseointegration [30, 31] but might only later undergo resorption and/or replacement by native bone in the natural cycle of bone-remodeling [32] .

When compared, both the minimal invasive transcrestal sinus lift and the subperiosteal tunnel technique provide a subperiosteal scaffold with the bony base of maxillary bone, which is filled then with devital (free) autologous, xenogeneic, or synthetic bone grafts (Figure 2) to create a sufficient future bone-volume and biomechanical stable crestal bone for dental implant insertion. The biomechanical properties of the restored alveolar crest (or subantral augmentation) and satisfactory long-term implant prognosis are expressed best by higher insertion-torque-values (ITVs) determined at dental implant insertion into the augmented alveolar crest (or subantral augmentation) compared to natural maxillary bone [33]. Since a successful restoration of the alveolar crest in the timely healing process after GBR is mandatorily preceded by sufficient angiogenesis and vascularization of the scaffold especially in critical size defects of more than $2.7 \mathrm{~mm}$ [34] the highly mechanosensitive nature of this process has to be taken into account strictly [35]: macro- and micromotions of the detached mucoperiosteum by muscular activities in and around the entire oral cavity, around the jawbones and inside the maxillary sinus by breathing activity cannot be avoided at all but might be alleviated by an enhancement of the preceding angiogenesis in the healing cycle. This suggested enhancement was attributed to Platelet Rich Plasma (PRP) [36] or, more recently, to Platelet Rich Fibrin (PRF) [37, 38] also for its properties of enhanced osteogenic differentiation of mesenchymal stem cells originating from the peri- and endosteum $[39,40]$.

The clinician in his daily routine work therefore has to rely on GBR-techniques, biomaterials, and transplant procedures that provide reliable and reproducible results with possible long-term dental implant success expressed by easily obtained and proven reliable clinical parameters such as the insertion-torque-value (ITV) at implant insertion [41-43].

Aim of the study was to investigate the resulting biomechanical stability of the restored alveolar crest after completed bone regeneration when two different moldable and in situ hardening calcium phosphate bone graft substitutes [30] are used with the subperiosteal tunnel technique in the anterior maxilla to determine if this new class of biomaterials, by their inherent physical property of demanded immobilization [35] of the augmentation scaffold, could achieve consistent results expressed by comparable or higher ITVs than natural maxillary bone and the influence of Platelet Rich Fibrin (PRF) [31] when added to the bone-block-like biomaterial. The results then were compared with the results of a similar study [33] investigating the mechanical quality of restored subantral bone using four different long-used and well documented biomaterials in transcrestal sinus lifting as to possibly demonstrate the suggested biological and clinical similarity between these two different surgical techniques. 


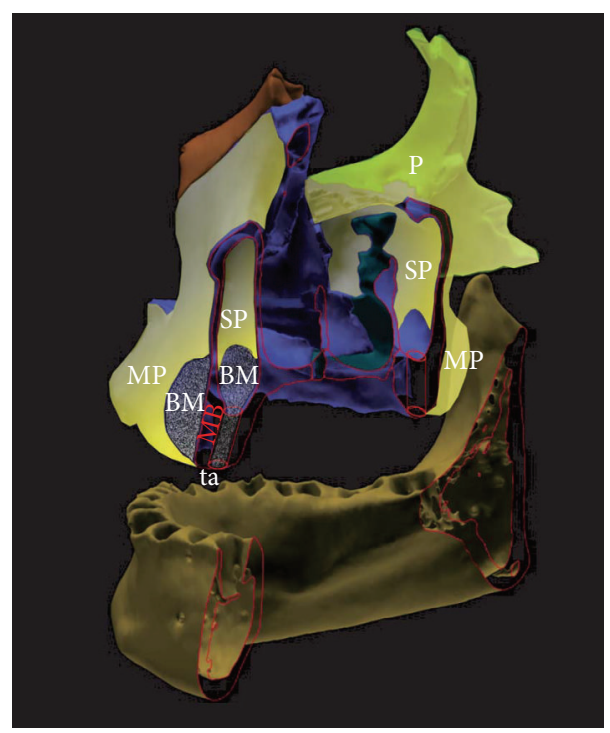

(a)

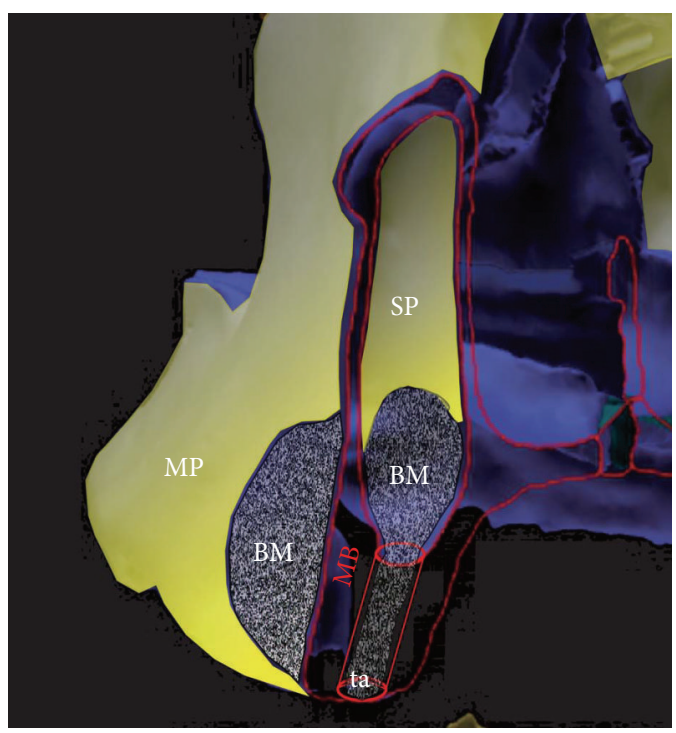

(b)

FIGURE 2: Graphical depiction of anatomical correlation and biological similarity of sinus lift versus buccal subperiosteal tunnel techniqueaugmentation-sites (overview (a), detail (b); periosteal cover of all bone surfaces depicted in yellow) MB: maxillary bone outlines (red) MP: mucoperiosteum of the oral cavity, SP: sinus periosteum of the maxillary sinus, P: periosteal cover of facial bones, BM: biomaterial applied subperiosteally in sinus lift and subperiosteal tunnel site, and ta: transcrestal approach of the tHUCSL-INTRALIFT-procedure.

\section{Material and Methods}

82 consecutive regular patients eligible for guided bone regeneration in the anterior maxilla from incisor to canine region with sufficient alveolar crest height of a minimum of $14 \mathrm{~mm}$ and less than $3 \mathrm{~mm}$ alveolar crest width were treated with the Piezotome enhanced subperiosteal tunnel technique (PeSPTT). The patients were aged between $29 \mathrm{yrs}$ and $71 \mathrm{yrs}$.

82 subperiosteal tunnel sites from the maxillary first incisor to the canine region were augmented in these patients and 109 implants were inserted after the healing period. All patients were antimicrobial shielded with either Amoxicillin/ Clavulan Acid 1 g $2 \times /$ day or Clindamycin $300 \mathrm{mg} 3 \times /$ day for 5 days, starting one day before surgery.

26 patients presenting an alveolar crest height of a minimum of $14 \mathrm{~mm}$ and minimum width of $5 \mathrm{~mm}$ served as control group of natural maxillary bone in the front-to-canine region receiving the same implants as the study groups (single stage Q1-Implant, 3.5/14 mm, TRINON Karlsruhe GmbH/ GER) resulting in 30 anterior maxillary implant sites $(n=30)$.

Since subperiosteal tunnel technique is a scientifically well-established and documented surgical procedure and the chosen biomaterials are certified in the European Union, no approval from an ethical committee was necessary according to EMEA guidelines for this clinical study. Every patient signed the consent to receive the CE-certified biomaterialvariant randomly as well as possibly advanced Platelet Rich Fibrin (aPRF), obtained from autologous blood.

To achieve a standardized reproducible clean separation of the intact periosteum from the bone (Figures 3 and 4 ) when creating the subperiosteal tunnel, an ultrasonic device was used (Piezotome II or Implantcenter II/Satelec-ACTEON/ France) for preparation with the BS 4-tip.

After clinical inspection (Figure 5) a vertical mucoperiosteal incision of approximately $10 \mathrm{~mm}$ was done $5-8 \mathrm{~mm}$ mesial of the augmentation site (Figure 6) followed by the preparation of the subperiosteal tunnel with the ultrasonic working-tip BS 4 (Figure 7(a)) attached to the hand piece of the Piezotome II or Implant Center II device (Figure 7(b)). The entrance of the tunnel then was checked for width (Figure 8) to allow insertion of the syringe with the biomaterial (Figure 9) and if assigned an additional layer of aPRFmembrane (Figure 10(a)). To achieve comparable and unbiased results $1 \mathrm{ccm}$ of biomaterial was applied per tooth site (i.e., $1 \mathrm{ccm}$ for single-tooth-gaps, $2 \mathrm{ccm}$ for a gap of two missing teeth, etc.) After molding and hardening of the biomaterial by natural blood flow or injection of aPRF-liquid the vertical incision was closed by single-stitch sutures (Figure 11).

The moldable and in situ hardening biomaterial as well as the addition of aPRF was randomly assigned (Excel Random Generator formula "=RUNDEN(ZUFALLSZAHL() $* 2$; $0)+1$ ") in three specifications:

(1) easy-graft CRYSTAL, granule size $0.45-1 \mathrm{~mm}$ (SUNSTAR Degradable Solutions AG/Zurich/CH): microporous compound particles of $40 \%$ beta-tricalcium phosphate (beta-TCP) and 60\% hydroxyapatite (HA), each particle covered by a 10 micrometer layer of polylactic-co-glycolic acid (PLGA); the primary loose particles are perfused with a "Biolinker" (N-methyl2-pyrrolidone solution) and once the Biolinker is washed out by the natural blood flow the biomaterial 

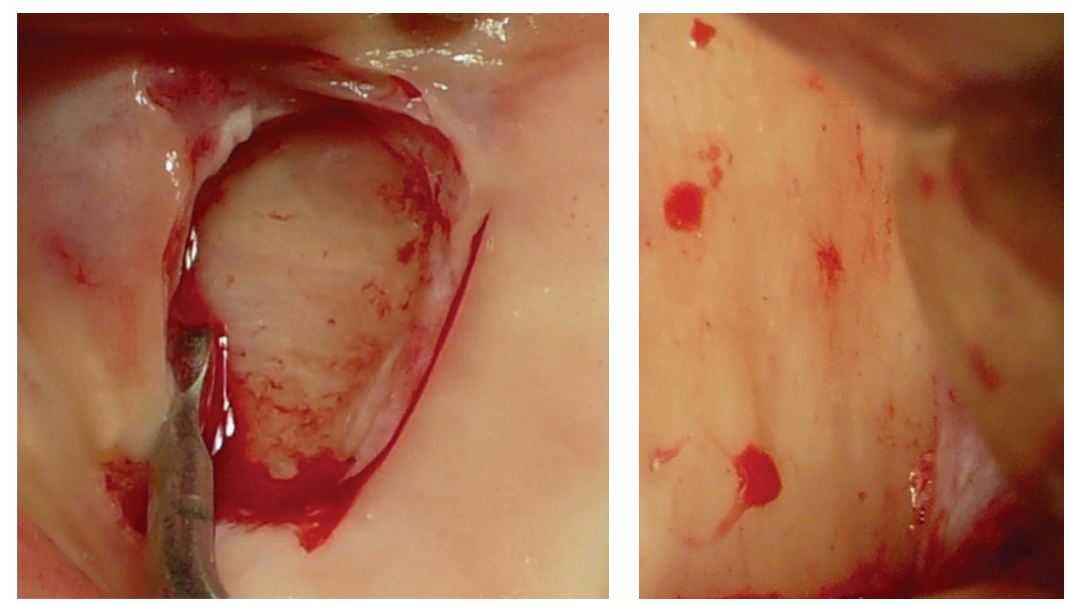

FIgURE 3: Macroscopical clinical demonstration of clean detachment of the periosteum from the bone with Piezotome surgery utilizing the cavitation effect: no remnants of the osteogenic layer of the periosteum are visible on the bone; the perforating blood vessels are cleanly cut.

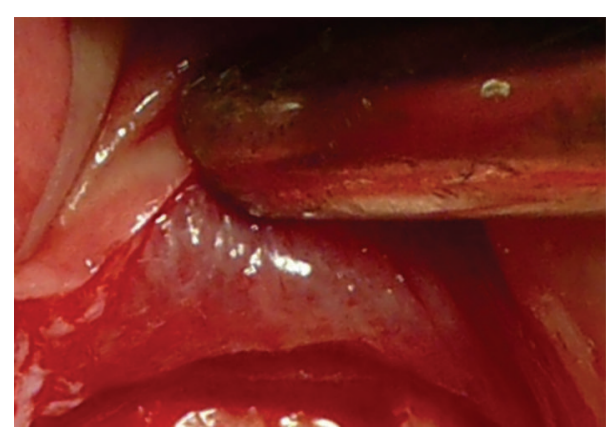

FIGURE 4: Close up view of the reverted periosteum cleanly detached from the bone with Piezotome surgery during preparation for a subperiosteal tunnel procedure. The intraperiosteal vessel-net is visible and undamaged.

hardens to a solid bone-substitute block (assigned implant sites: $n=36$ );

(2) easy-graft CLASSIC, granule size $0.5-1 \mathrm{~mm}$ (SUNSTAR Degradable Solutions AG/Zurich/CH): microporous particles of pure beta-tricalcium phosphate (beta-TCP), each particle covered by a 10 micrometer layer of polylactic-co-glycolic acid (PLGA); the primary loose particles are perfused with a "Biolinker" ( $\mathrm{N}$-methyl-2-pyrrolidone solution) and once the Biolinker is washed out by the natural blood flow the biomaterial hardens to a solid bone-substitute block (assigned implant sites: $n=35$ );

(3) easy-graft CLASSIC, granule size $0.5-1 \mathrm{~mm}$ (SUNSTAR Degradable Solutions AG/Zurich/CH): microporous particles of pure beta-tricalcium phosphate (beta-TCP), each particle covered by a 10 micrometer layer of polylactic-co-glycolic acid (PLGA); the primary loose particles are perfused with a "Biolinker" (N-methyl-2-pyrrolidone solution). Instead of washing out the Biolinker by natural blood flow the Biolinker was washed out forcefully with the liquid obtained

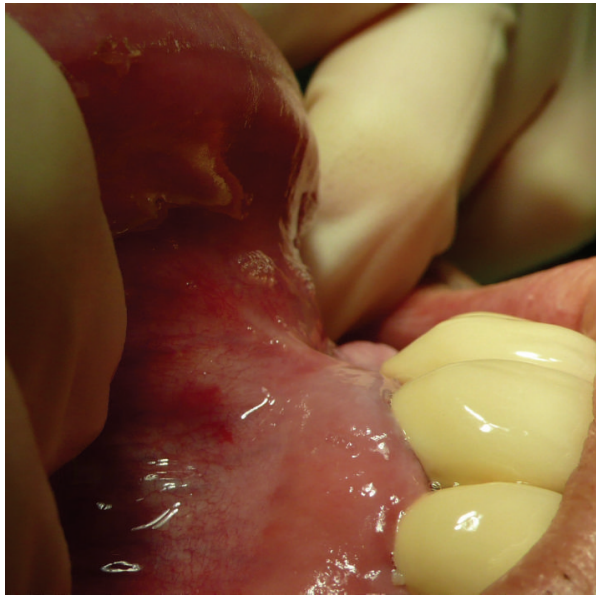

FIGURE 5: Presurgical clinical inspection of the lateral atrophic alveolar ridge.

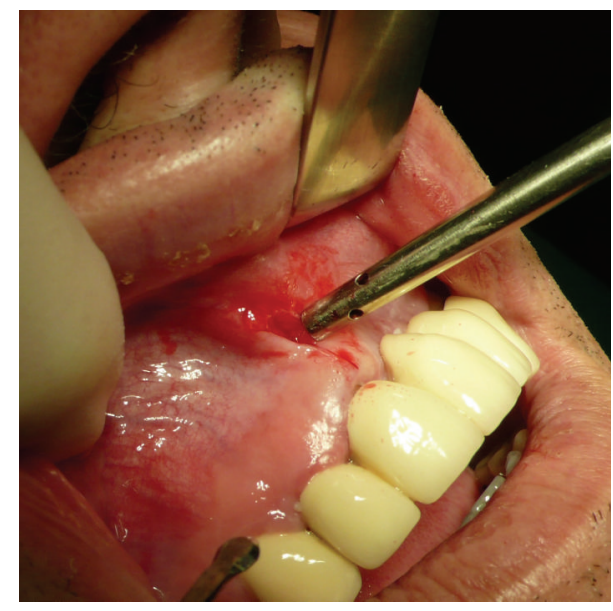

FIgURE 6: Vertical incision mesial of the augmentation site (left 1st and 2 nd incisor). 


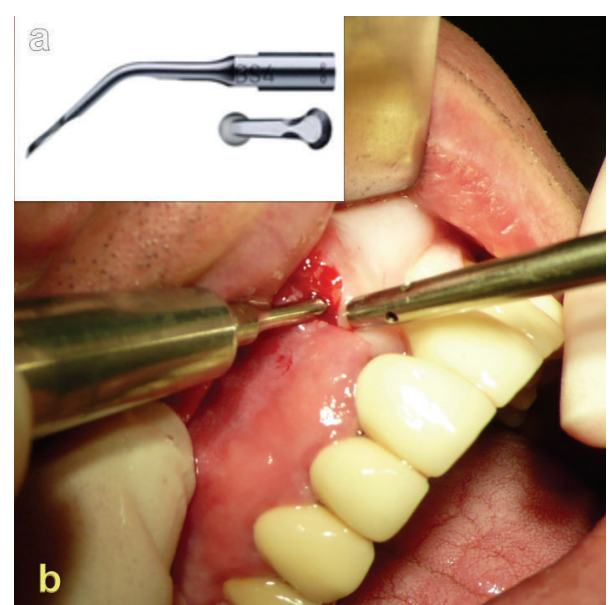

Figure 7: (a) BS-4 working tip for Piezotome II/SOLO/Implant Center II. (b) Clean detachment of the periosteum from the maxillary bone with the Piezotome-device up to the basal margins of the nasal cavity.

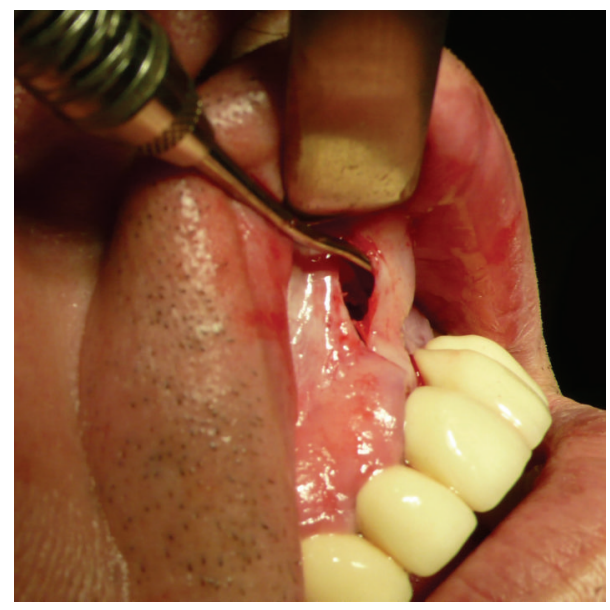

FIGURE 8: Inspection of the subperiosteal tunnel and probe-testing for sufficient pocket-extensions to the left canine-region.

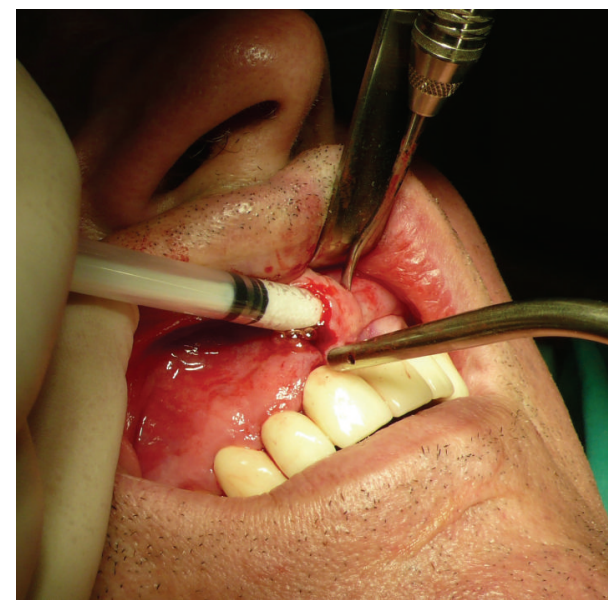

FIGURE 9: Insertion of the moldable and in situ hardening biomaterial.

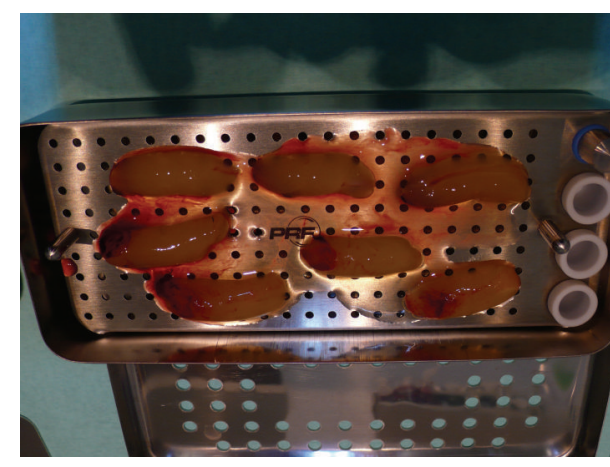

(a)

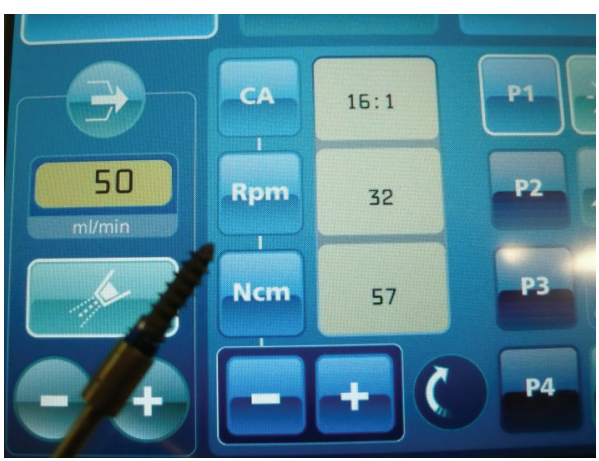

(b)

FIgURe 10: (a) Preparation of PRF to be applied to pure bTCP in situ hardening biomaterial when randomly assigned. The PRF-clots will be compressed to form an elastic membrane and the expressed liquid aspirated with a sterile syringe for later washout of the "Biolinker" to harden the biomaterial to a bone-block-like graft. By this procedure active angiogenic and osteoblast stimulating cells are perfused inside the hardened porous biomaterial block. (b) Torque measurement in $1 \mathrm{Ncm}$-steps with the Implantcenter II, implant motor.

in the preparation of advanced Platelet Rich Fibrin membranes (aPRF/SYFAC/France) in a sterile syringe to harden the biomaterial and a layer of aPRF was placed subperiosteal (assigned implant sites: $n=38$ ).

After 6-7 months a control CBCT of the augmentation site was taken to check the bone dimensions for the planned implant insertion (Figure 12).

Following the results of a study investigating the average time for completion of bone-regeneration in sinus lift procedures [6] and to achieve an unbiased comparison with the results obtained in the sinus lift study [33] implants were inserted after an average healing time of 8.3 months (max: 8.6 months, min: 7.9 months).

In all cases one, two or three single stage Q1-implants (self-taper, root analogue screw-design; TRINON-Karlsruhe $\mathrm{GmbH} / \mathrm{GER}$ ) with uniform dimensions of $14 \mathrm{~mm}$ length and diameter $3.5 \mathrm{~mm}$ were inserted into the augmentation site after a top crestal incision and minimal reversion of the mucoperiosteal flaps to the buccal and palatal side for clinical inspection of the alveolar crest (Figures 13, 14(a), and 14(b)). To achieve unbiased results the Q1-implants (Figure 14(a)) 


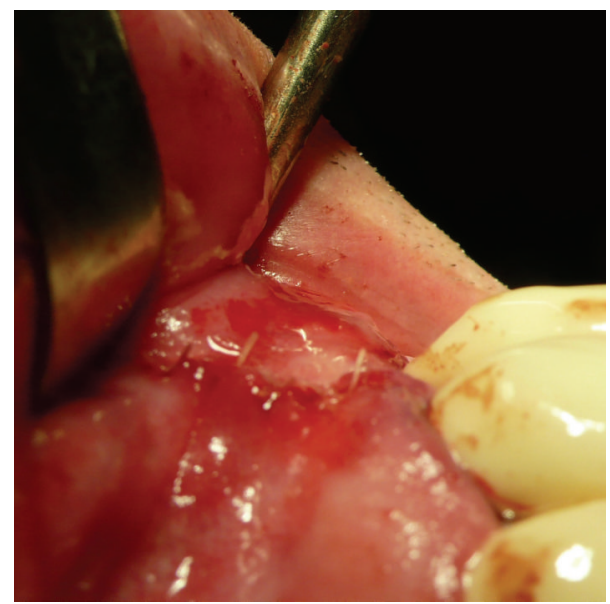

FIGURE 11: Wound closure with single stitches.

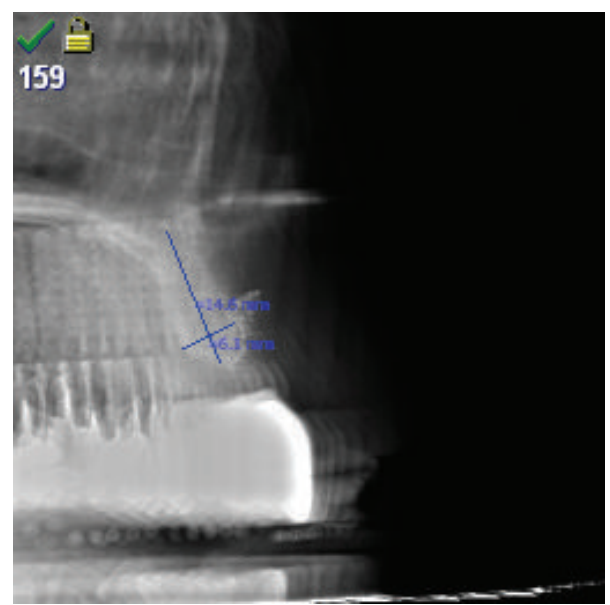

FIGURE 12: CBCT control and measurement of achieved bone dimensions prior to implant insertion.

were inserted by a different surgeon than the surgeon performing the subperiosteal tunnel procedure and randomly assigning the biomaterial.

All implants were inserted precisely following the drilling protocol required by the Q1-implant manufacturer with pilot and final form drilling at $50 \mathrm{rpm}$ with unused drills for each implant. No specimens for histologic analysis could have been taken with a trephine-drill due to the conical shape of the final form-drill for the single-stage Q1-implant to prevent biases of ITV-measurements.

Determination of insertion-torque-value (ITV) was taken then with the Implantcenter II (Satelec-ACTEON/FR) for each inserted implant, allowing a torque increase in steps of $1 \mathrm{Ncm}$ up to $100 \mathrm{Ncm}$ (Figure 10(b)). Implant insertion was started with a basic torque-setting of $15 \mathrm{Ncm}$ increasing in $1 \mathrm{Ncm}$-steps by an assistant until the single stage Q1-implant was inserted to its full length of $14 \mathrm{~mm}$. All implants were treated with provisional, anatomical correct resin-crowns with no occlusal contacts for a period of 3 months.

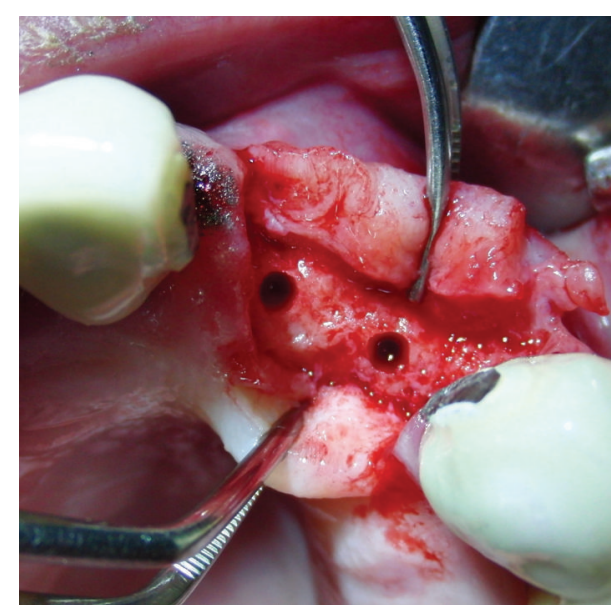

FIGURE 13: Crestal opening of the augmentation site, clinical inspection, and pilot- and form-drilling for implant insertion.

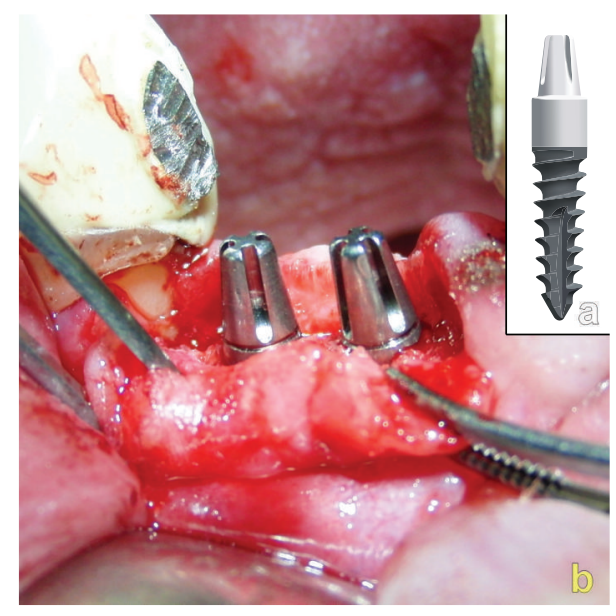

FIGURE 14: (a) Q1-Implant shape. (b) Q1-implants with a diameter of $3.5 \mathrm{~mm}$ and length of $14 \mathrm{~mm}$ inserted into the augmentation site.

Statistical evaluation was performed by a one-way ANOVA-test, Student's $t$-test, and post hoc multiple comparisons by Fisher's least significant difference (LSD) to test mean insertion-torque-values and variance in each group and mean difference significance between all groups. Additionally, the data were depicted in notched box plots to show the data distribution and interquartile ranges (IQR) between the 25th and 75th percentile of the specific biomaterial tested.

The results then were compared with the results of a prior similar study investigating drill-torque and insertion-torquevalues after sinus lifting with the tHUCSL-INTRALIFT-method using four different biomaterials (Bio-Oss, NanoBone, easy-graft CLASSIC, easy-graft CRYSTAL) [33]. The basic setup and healing periods for this study were the same and by this unbiased comparable to the present study. The slightly different dimension of the two-stage Q2-implant used in the prior study is compensated mathematically by a $2 \mathrm{~mm}$ longer length of the single-stage Q1-implant (Figure 15). 


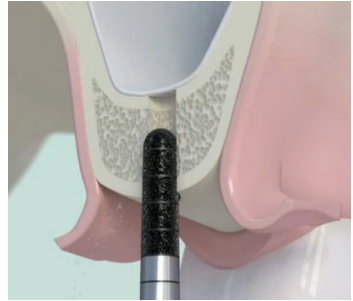

(a)

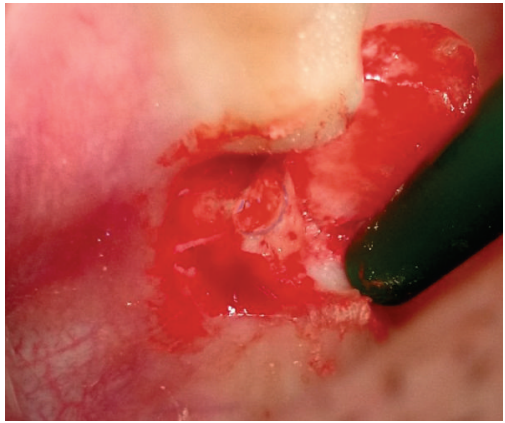

(e)

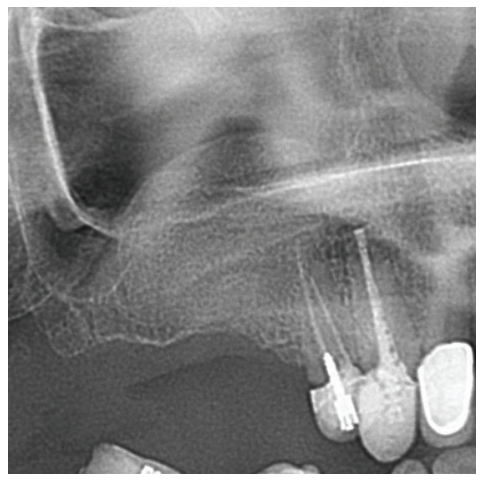

(h)

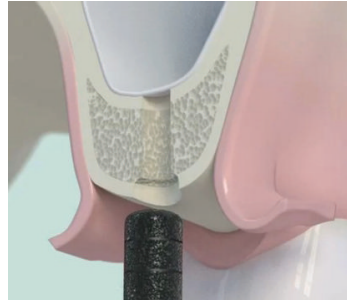

(b)

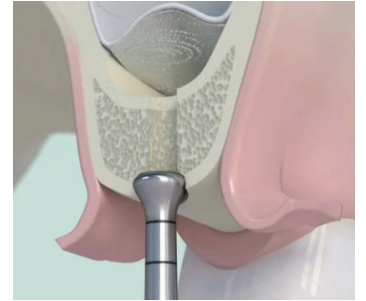

(c)

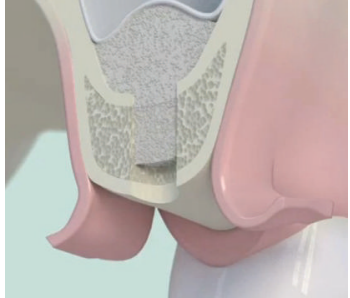

(d)

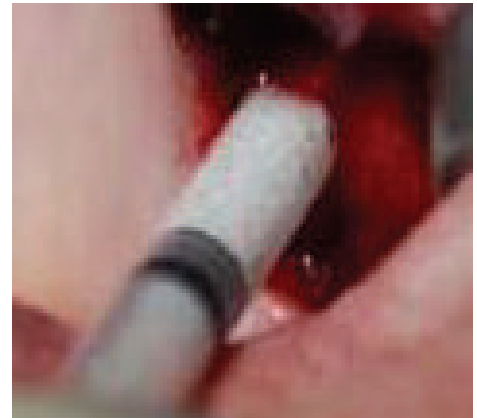

(f)

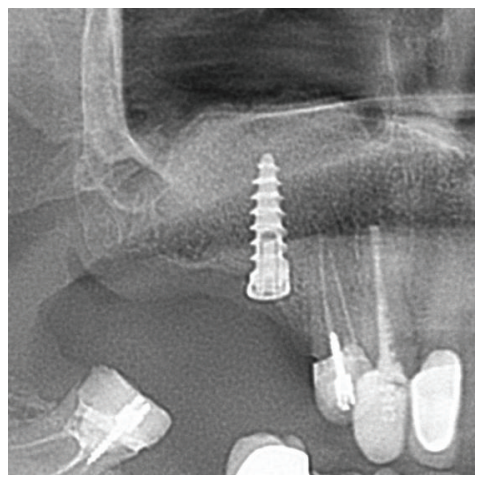

(i)

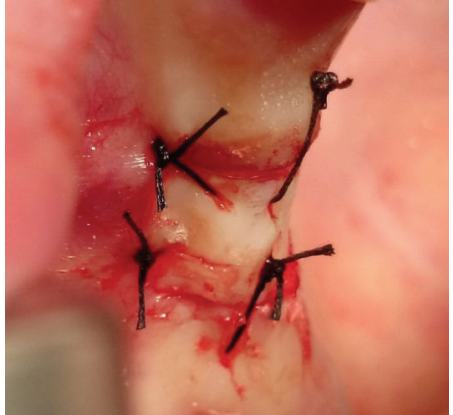

(g)

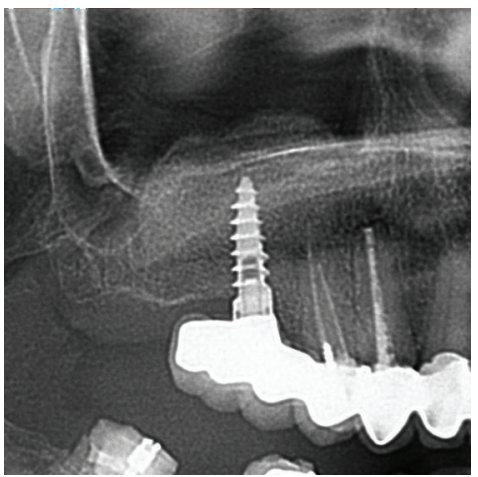

(j)

FIGURE 15: Overview tHUCSL-INTRALIFT procedure with Piezotome II/SOLO: (a) after reverting a minimal invasive crestal flap the sinus floor is opened transcrestal with the diamond coated Piezotome-tip TKW2, (b) a sealing receptacle is prepared with the diamond-coated Piezotome-tip TKW4, (c) the Piezotome-tip TKW5 is tight-fit inserted into the receptacle to prevent liquid-back-flow to the oral cavity and by this subantral loss of hydrodynamic pressure. The Piezotome then is activated and the periosteum of the Schneiderian membrane cleanly detached from the bony antrum floor utilizing the cavitation effect. (d) Insertion of $2 \mathrm{ccm}$ biomaterial into the subperiosteal scaffold, (e) clinical case of a typical tHUCSL-INTRALIFT-procedure, (f) subantral application of $2 \mathrm{ccm}$ biomaterial, (g) wound closure, (h) postsurgical $\mathrm{X}$-ray control of the sinus lift site, (i) implant insertion after average 8.7 months, and (j) final prosthetic treatment.

\section{Results}

All surgeries proceeded uneventful with no complications such as infections or wound dehiscences. Patients reported only minor swellings during the first 5 days postsurgical and almost no pain (average intake of Ibuprofen $400 \mathrm{mg}$ mean value: 3.2 tablets, max: 6 tablets, min: 0 tablets). The patient's main complaint referred to a "voluminous feeling" beneath the lip and under the nose in the augmentation area, especially when no interim bridge was attached as preexistent or newly manufactured provisional restoration but only removable partial overdentures without any buccal resin shield.

All subperiosteal tunnel augmentation sites presented sufficient even buccal bone gain in radiographic follow-up prior to implant insertion with a minimum top-crestal ridge width of $6 \mathrm{~mm}$.

Insertion-torque-values (ITV) were lowest in the control group when implants were inserted into native anterior maxillary bone (mean value: $27.87 \mathrm{Ncm}$, stand. dev.: $6.66 \mathrm{Ncm}$ ) (Figure 16) but significantly $(P<0.05)$ higher when compared to native maxillary bone in the premolar and molar region (mean value: $22.11 \mathrm{Ncm}$, stand. dev.: $4.64 \mathrm{Ncm}$ ) [33] (Figure 17).

ITVs were significantly $(P<0.05)$ different between all groups and highest (mean value: $52.5 \mathrm{Ncm}$, stand. dev.: $8.15 \mathrm{Ncm}$ ) in the group treated with the biphasic self-hardening biomaterial (BiSHB: 60\% HA, 40\% bTCP) followed by 


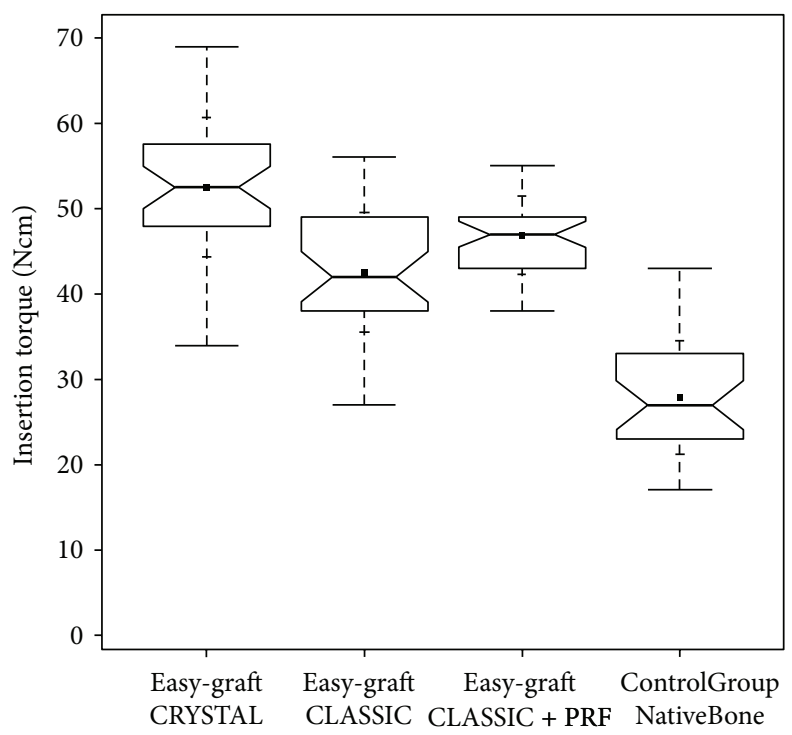

FIGURE 16: Insertion-torque-value (ITV) comparison notched box plot for the subperiosteal tunnel procedure in the anterior maxilla depicts the interquartile range (IQR) between the 25th and 75th percentile of the specific biomaterial tested where 50\% of the data points were located. Additionally, the upper whiskers represent data within the 75 th percentile +1.5 times the IQR. The lower whisker delimits data of the 25th percentile -1.5 times the IQR. Within the boxes the notches mark the confidence interval based on the median \pm 1.58 (IQR/sqrt of $n$ ) ( $n$ = number of measurements). Additionally, the mean value is indicated by a black square and the cross symbol (+) displays the standard deviation. Easy-graft CRYSTAL $(n=36)$, easy-graft CLASSIC $(n=35)$, easy-graft CLASSIC + Platelet Rich Fibrin $(n=38)$, and control group native anterior maxillary bone $(n=30)$.

the group treated with monophasic self-hardening biomaterial (MoSHB: 100\% bTCP) with addition of advanced Platelet Rich Fibrin (aPRF) (mean value: $46.89 \mathrm{Ncm}$, stand. dev.: $4.57 \mathrm{Ncm}$ ) and without addition of aPRF (mean value: $42.51 \mathrm{Ncm}$, stand. dev.: $7.03 \mathrm{Ncm}$ ) and the control group of native bone (mean value: $27.87 \mathrm{Ncm}$, stand. dev.: $6.66 \mathrm{Ncm}$ ) (Table 1 and Figure 16).

Comparing the ITVs for BiSHB obtained with the Piezotome-enhanced subperiosteal-tunnel-technique (PeSPTT) in the anterior maxilla with the results of the tHUCSLINTRALIFT study [33] the mean ITV values in the INTRALIFT group were significant $(P<0.05)$ higher (INTRALIFT group: mean value: $56.58 \mathrm{Ncm}$, stand. dev.: $3.36 \mathrm{Ncm}$, PeSPTT group: mean value: $52.5 \mathrm{Ncm}$, stand. dev.: $8.15 \mathrm{Ncm})$ and standard deviation significant $(P<0.05)$ lower (INTRALIFT group: stand. dev.: $3.36 \mathrm{Ncm}$ versus PeSPTT group: stand. dev.: $8.15 \mathrm{Ncm}$ ) (Table 2 and Figure 18).

Contrary the ITVs for MoSHB with addition of aPRF were higher when used for the Piezotome-enhanced subperiosteal-tunnel-procedure in the anterior maxilla (mean value: $46.89 \mathrm{Ncm}$ ) compared to MoSHB without aPRF applied to INTRALIFT sites (mean value: $45.85 \mathrm{Ncm}$ ), but not significant $(P=0.41)$ as well as for standard deviation (PeSPTT sites: $4.57 \mathrm{Ncm}$ versus INTRALIFT-sites: $5.01 \mathrm{Ncm}$ ) (Table 2 and Figure 18).

MoSHB without aPRF applied to PeSPTT sites revealed a significant $(P=0.01)$ lower ITV (mean value: $42.51 \mathrm{Ncm}$ ) compared to MoSHB applied to INTRALIFT sites (mean value: $45.85 \mathrm{Ncm}$ ) with a higher standard deviation (SP: $7.03 \mathrm{Ncm}$ versus IL: $5.01 \mathrm{Ncm}$ ) (Table 2 and Figure 18).
In general, standard deviation for both BiSHB and MoSHB without aPRF in PeSPTT-sites (SP) was significantly higher than in INTRALIFT (IL) sites and subperiosteal tunnel sites (SPPRF) treated with MoSHB with aPRF (SP-BiSHB: $8.16 \mathrm{Ncm}$ and SP-MoSHB: $7.03 \mathrm{Ncm}$ versus ILBiSHB: $3.6 \mathrm{Ncm}$, IL-MoSHB: $5.01 \mathrm{Ncm}$, and SPPRF-MoSHB: $4.57 \mathrm{Ncm}$ ) (Figure 18).

An overall cumulative comparison between the tested loose granule biomaterials (bovine bone: BioOss, synthetic graft: Nanobone $100 \% \mathrm{HA}$ embedded in a $\mathrm{SiO}_{2}$-matrix) for the INTRALIFT procedure and MoSHB and BiSHB applied in both INTRALIFT and PeSPTT procedures (Table 2) is depicted in Figure 19. No statistical significant differences were found when the ITV of the control group in the anterior maxilla are compared with INTRALIFT sites augmented with bovine bone $(P=0.2)$ and MoSHB + aPRF in PeSPTT sites with INTRALIFT sites augmented with MoSHB without $\operatorname{aPRF}(P=0.4)$.

\section{Discussion}

The physiological cycle of bone remodeling is a continuous event and is adjusted by peri- and endosteum [44]. Bone mass and mechanical strength increases on continuous demand to bear higher loads or decreases down to atrophy by lack of physiological load as given in an edentulous alveolar crest or general or bone-specific diseases [32]. Muscles, tendons, teeth, periosteum, and endosteum are intimately connected to and anchored in the calcified structure of bone by elastin-rich collagenous Sharpey fibres which "integrate into 


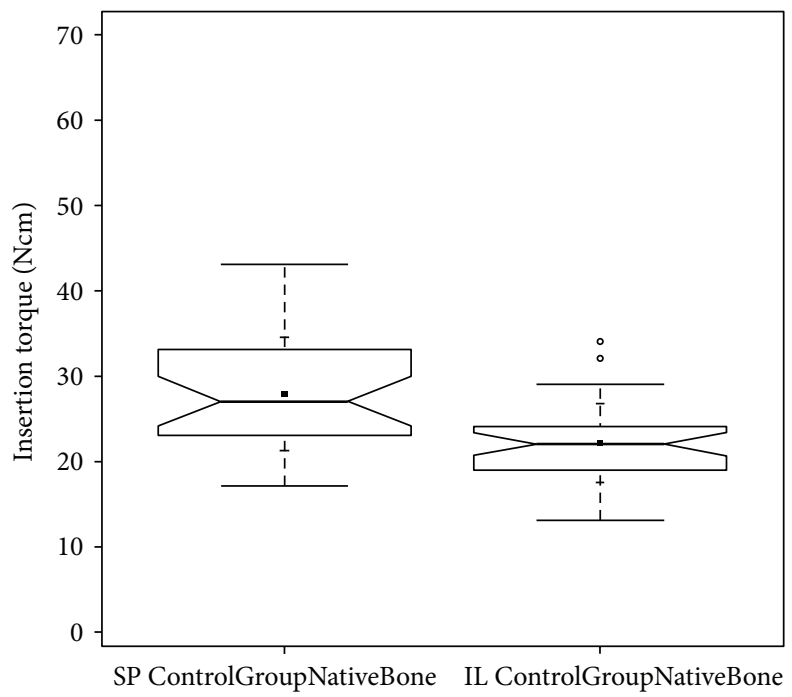

FIGURE 17: ITV-comparison notched box plot of native anterior maxillary bone (subperiosteal tunnel procedure control group-SP ControlGroupNativeBone) and native molar maxillary bone (INTRALIFT control group-IL ControlGroupNativeBone) depicts the interquartile range (IQR) between the 25th and 75th percentile of the specific biomaterial tested where 50\% of the data points were located. Additionally, the upper whiskers represent data within the 75 th percentile +1.5 times the IQR. The lower whisker delimits data of the 25 th percentile -1.5 times the IQR. Within the boxes the notches mark the confidence interval based on the median $\pm 1.58(\mathrm{IQR} / \mathrm{sqrt}$ of $n)(n=$ number of measurements). Additionally, the mean value is indicated by a black square and the cross symbol $(+)$ displays the standard deviation. Control group native anterior maxillary bone SP $(n=30)$, control group native molar maxillary bone IL $(n=35)$.

a periosteal Sharpey fiber-endosteum (PSE) structural continuum" allowing muscular forces to act on bone in its function as lever for movement withstanding static and dynamic loading forces. The same principle is valid for the elastin-rich Sharpey fibres of the periodontal ligament that interweaves the entire alveolar ridge [45]. The maintenance of the alveolar ridges is the result of functional loaded teeth within its "biological load width" and not its precondition.

The elastin-rich Sharpey fibres too are most suitable to distribute and dampen the strain on the calcified structures of bone. This "periosteal Sharpey fiber-endosteum (PSE) structural continuum" together with the connected "acting" organs (muscles, tendons, and teeth) shows an efficient organizational pattern relative to forces introduced. By this, Sharpey fibres are suggested as main peri- and endosteal trigger for increase of bone mass and biomechanical stability on continuous higher loads by stimulation of osteoblasts originating from the cambium layer of peri- and endosteum [45]. Contrary, a lack of forces introduced into bone by the PSEstructural continuum (as a reaction to physical inactivity or in extreme, e.g., during spaceflights by the lack of gravity) leads to disorganization of the Sharpey fibres followed by a decrease of calcified bone mass down to atrophy $[46,47]$ proving the vital role of peri- and endosteum in bone maintenance, repair, and regeneration. Chronic overload exceeding the "biological biomechanical resistance width" leads to bone resorption and possible loss of osseointegrated (dental) implants too but is used therapeutically in orthodontics. Compared to long bones the mandible contains a greater amount of collagen and by its structural specifics "likely renders more flexibility to the bone and leaves it more suited to constant exercise," remodeling and higher load bearing [48] which might probably be supportive for GBR procedures in CMF and oral surgery.

But also among the jawbones, the maxilla and the mandible, significant differences can be found: the mandibular alveolar crest provides a 2.8-fold greater bone volume compared to the maxillary alveolar crest and shows a significant and, regarding anterior and posterior sections, uniformly higher bone formation rate compared to the maxilla, whereas in the maxillary alveolar crest significant differences of bone formation can be found in the anterior section compared to the posterior maxilla [18]. These experimental results obtained in an animal experiment can be backed now clinically in humans when the ITVs of natural subantral bone are compared with ITVs in the anterior maxilla (Figure 17).

While the PSE structural continuum mainly maintains crestal bone and induces bone regeneration, surgical GBR techniques, mostly creating "critical size defects" far above the biological threshold [33], demand a proper immobilization beyond the biological threshold [29] of the augmented scaffold in toto to allow the first step in bone restoration: proper vascularization [35] and osseointegration of biomaterials [30] and/or implants. This mandatory immobilization of the augmentation scaffold to prevent fibrous tissue encapsulation of the implanted grafts [27-29] can be obtained by traditional surgical methods: free bone block grafts, harvested from the chin or lateral ramus of the mandible, are rigidly fixed to the augmentation site with osteosynthesis screws. These procedures need the preparation of vast full thickness mucoperiosteal flaps and by this the partial destruction of the periosteum and its vascularization by periosteal slotting to achieve a tensionless wound closure. Vascularization of the augmentation site is massively obstructed by the surgical 
TABLE 1: Statistical evaluation of insertion-torque-values obtained at implant insertion in augmented sites of the anterior maxilla (subperiosteal tunnel technique $\{\mathrm{SP}\}$ ) by one-way ANOVA test, Student's $t$-test, and post hoc multiple comparisons by Fisher's least significant difference (LSD) to test mean insertion-torque-values and variance in each group and mean difference significance between all groups.

(a)

\begin{tabular}{lr}
\hline Means comparisons & \\
\hline Comparisons for each pair using Student's $t$ & Confidence quantile \\
\hline$t$ & \\
\hline 1.97769 & Alpha \\
\hline
\end{tabular}

(b)

\begin{tabular}{|c|c|c|c|c|}
\hline \multicolumn{5}{|l|}{$\begin{array}{l}\text { LSD threshold matrix } \\
\text { Abs(Dif)-LSD }\end{array}$} \\
\hline & $\begin{array}{c}\text { Easy-graft } \\
\text { CRYSTAL_SP }\end{array}$ & $\begin{array}{c}\text { Easy-graft } \\
\text { CLASSIC + } \\
\text { PRF_SP }\end{array}$ & $\begin{array}{l}\text { Easy-graft } \\
\text { CLASSIC_SP }\end{array}$ & $\begin{array}{l}\text { Control Group Native Bone } \\
\text { Front-Canine_SP }\end{array}$ \\
\hline Easy-graft CRYSTAL_SP & -3.12 & 2.52 & 6.84 & 21.36 \\
\hline Easy-graft CLASSIC + PRF_SP & 2.52 & -3.04 & 1.28 & 15.79 \\
\hline Easy-graft CLASSIC_SP & 6.84 & 1.28 & -3.17 & 11.35 \\
\hline $\begin{array}{l}\text { ContrGroupNativeBone } \\
\text { Front-Canine_SP }\end{array}$ & 21.36 & 15.79 & 11.35 & -3.42 \\
\hline
\end{tabular}

Positive values show pairs of means that are significantly different.

(c)

\begin{tabular}{|c|c|c|c|c|}
\hline \multicolumn{5}{|c|}{ Connecting letters report } \\
\hline level & Mean & & & \\
\hline Easy-graft CRYSTAL_SP & $\mathrm{A}$ & & & 52.50 \\
\hline Easy-graft CLASSIC + PRF_SP & & B & & 46.89 \\
\hline Easy-graft CLASSIC_SP & & & & 42.51 \\
\hline ContrGroupNativeBone Front-Canine_SP & & & $\mathrm{D}$ & 27.87 \\
\hline
\end{tabular}

Levels not connected by same letter are significantly different.

(d)

\begin{tabular}{|c|c|c|c|c|c|c|c|}
\hline \multicolumn{8}{|c|}{ Ordered differences report } \\
\hline Level & $\begin{array}{l}\text { compared } \\
\text { to }\end{array}$ & Level & Difference & Std. err. dif. & Lower CL & $\begin{array}{l}\text { Upper } \\
\text { CL }\end{array}$ & $P$ value \\
\hline Easy-graft CRYSTAL_SP & & $\begin{array}{l}\text { ContrGroupNativeBone } \\
\text { Front-Canine_SP }\end{array}$ & 24.63 & 1.66 & 21.36 & 27.91 & $<0.0001$ \\
\hline Easy-graft CLASSIC + PRF_SP & & $\begin{array}{l}\text { ContrGroupNativeBone } \\
\text { Front-Canine_SP }\end{array}$ & 19.03 & 1.64 & 15.79 & 22.27 & $<0.0001$ \\
\hline Easy-graft CLASSIC_SP & & $\begin{array}{l}\text { ContrGroupNativeBone } \\
\text { Front-Canine_SP }\end{array}$ & 14.65 & 1.67 & 11.35 & 17.95 & $<0.0001$ \\
\hline Easy-graft CRYSTAL_SP & & easy-graft CLASSIC_SP & 9.99 & 1.59 & 6.84 & 13.13 & $<0.0001$ \\
\hline Easy-graft CRYSTAL_SP & & easy-graft CLASSIC + PRF_SP & 5.61 & 1.56 & 2.52 & 8.69 & 0.0005 \\
\hline Easy-graft CLASSIC + PRF_SP & & easy-graft CLASSIC_SP & 4.38 & 1.57 & 1.27 & 7.49 & 0.0060 \\
\hline
\end{tabular}

procedure itself. A high risk of multiple complications such as voluminous postsurgical edema (which by itself diminishes blood circulation in the surgical site), total, or partial resorption of the graft by lack of vascularization, wound dehiscence, and donor-site complications are commonly known to accompany these procedures.

Contrary, the results of the present and similar prior studies [31, 33, 37-39] suggest minimal invasive surgical procedures such as the tHUCSL-INTRALIFT [7] or the subperiosteal-tunnel-technique (SPTT) to preserve the functional integrity of the periosteum $[6,7,33]$ (Figures 1 and 3 ).

Obviously the use of xenogeneic bovine, synthetic HA and the new class of moldable, and self-hardening bone grafts (monophasic bTCP $\{\mathrm{MoSHB}\}$ or biphasic HA/bTCP $\{\mathrm{BiSHB}\})$ in guided-bone-regeneration procedures significantly enhances the (bio)mechanical stability of the restored anterior and subantral maxillary alveolar crest (Figure 19), but by a twofold when BiSHB is used. 


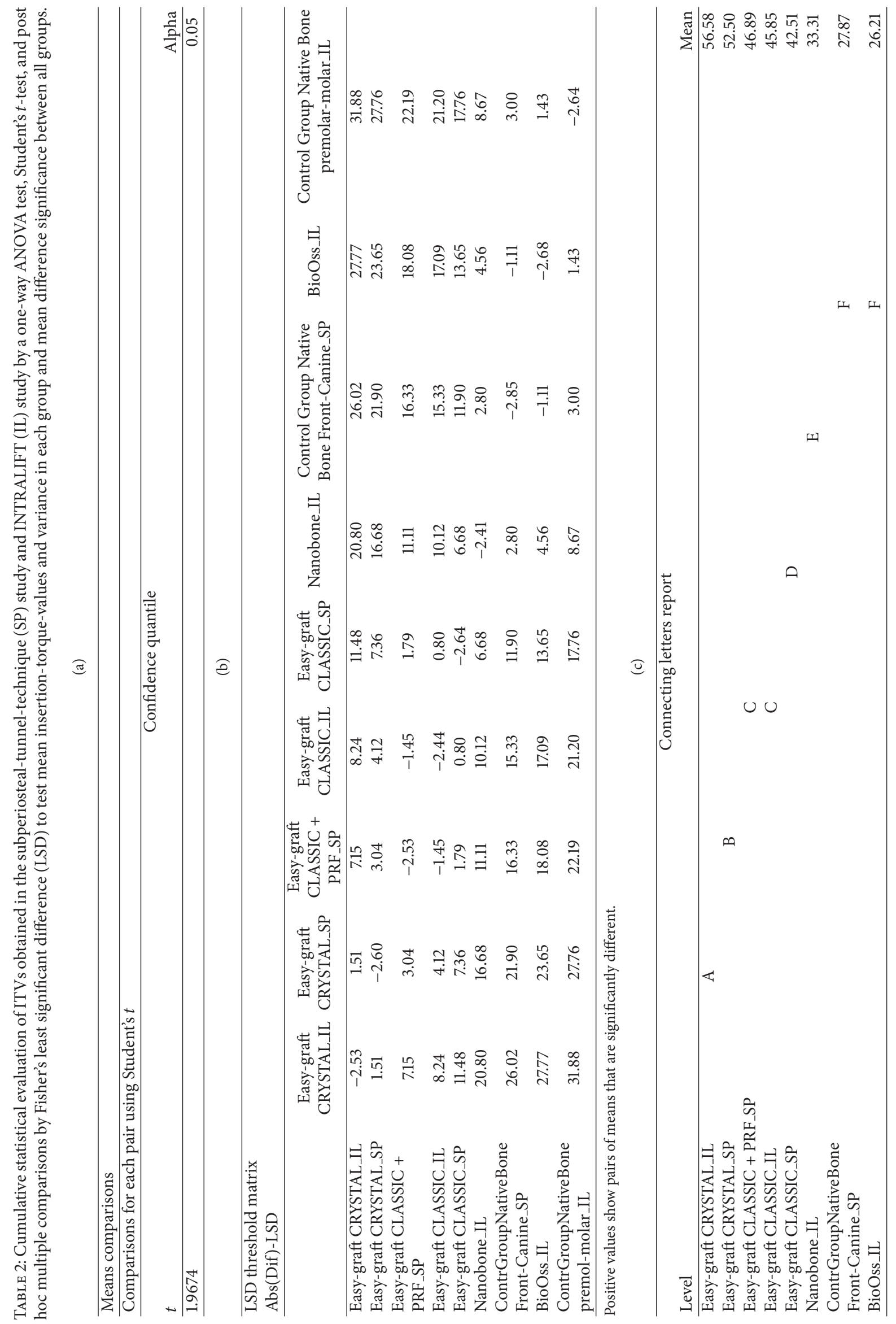




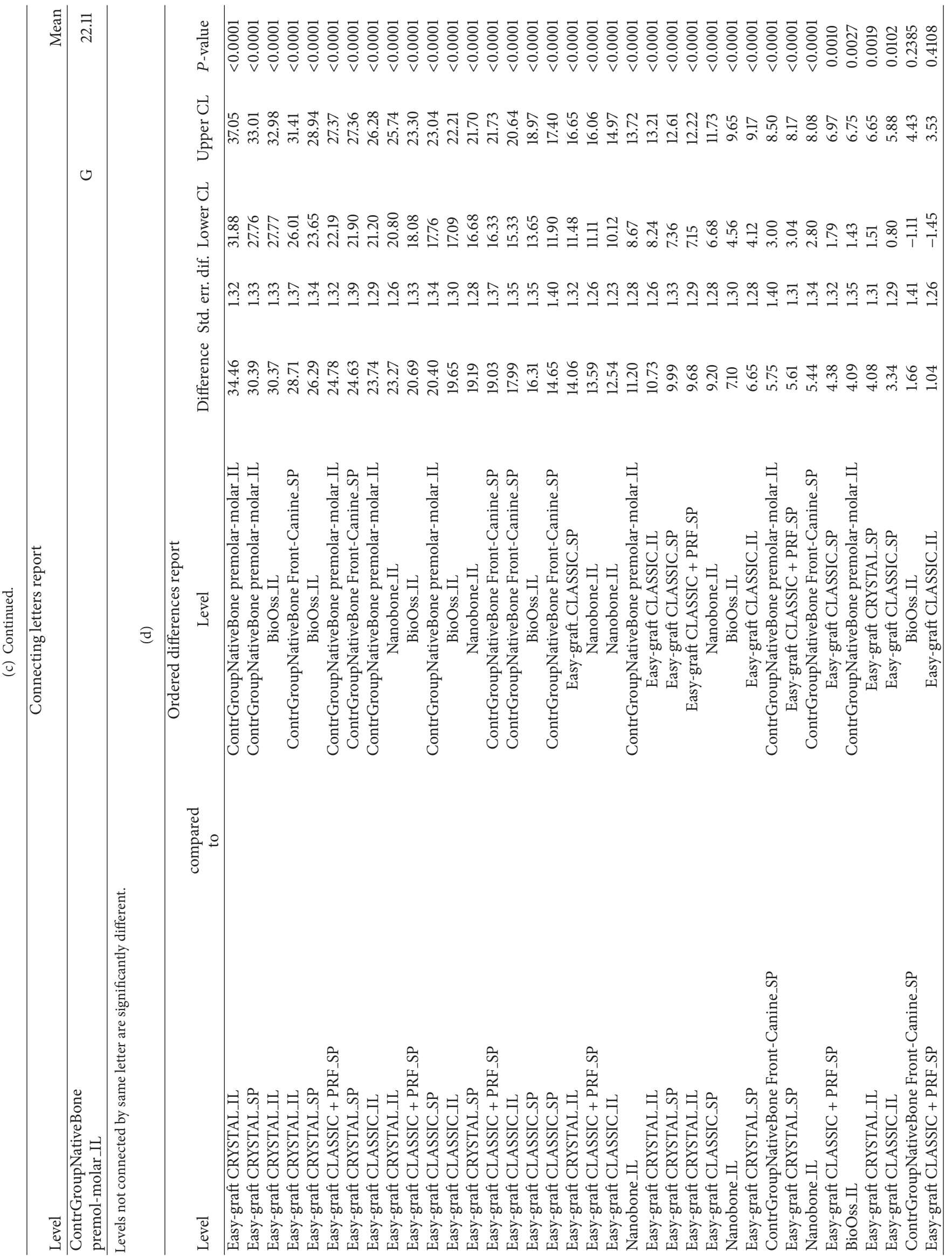




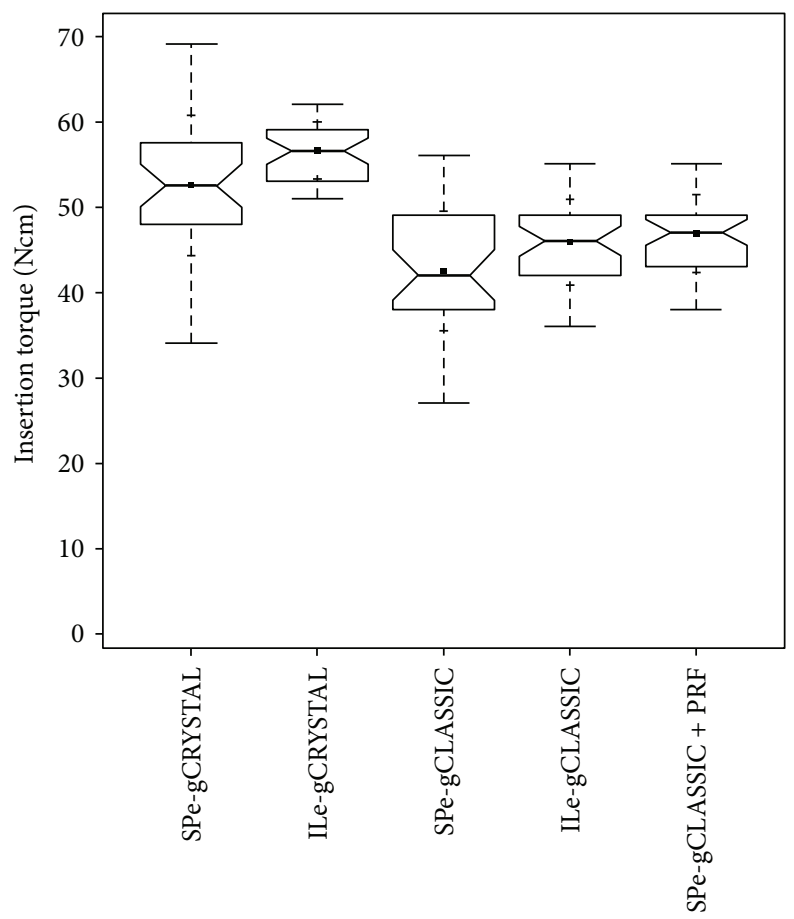

FIGURE 18: ITV-comparison notched box plot of the subperiosteal tunnel procedure (SP) in the anterior maxilla and the INTRALIFTprocedure (IL) with BiSHB (e-gCRYSTAL), MoSHB (e-gCLASSIC), and MoSHB + Platelet Rich Fibrin (PRF) (e-gCLASSIC + PRF) depicts the interquartile range (IQR) between the 25th and 75th percentile of the specific biomaterial tested where $50 \%$ of the data points were located. Additionally, the upper whiskers represent data within the 75 th percentile +1.5 times the IQR. The lower whisker delimits data of the 25th percentile -1.5 times the IQR. Within the boxes the notches mark the confidence interval based on the median \pm 1.58 (IQR/sqrt of $n$ ) ( $n$ = number of measurements). Additionally, the mean value is indicated by a black square and the cross symbol $(+)$ displays the standard deviation. SPe-gCRYSTAL $(n=36)$, ILe-gCRYSTAL $(n=38)$, SPe-gCLASSIC $(n=35)$, ILe-gCLASSIC $(n=41)$, and SPe-grCLASSIC + PRF $(n=38)$.

Contrary to native subantral maxillary bone compared to the anterior alveolar crest, the resulting (bio)mechanical stability after augmentation with BiSHB (60\% HA/40\% bTCP) and MoSHB (100\% bTCP), expressed by higher ITVs, reveals to be significantly higher with a significant lesser variance in tHUCSL-INTRALIFT sites compared to the subperiostealtunnel-augmentations in the anterior maxilla (Figure 18). Having the same maxillary bone-base and a comparable geometrical subperiosteal scaffold containing comparable volumes of BiSHB or MoSHB (Figure 2) the ITVs in the anterior maxilla should be at least equal to the tHUCSLINTRALIFT sites if not higher because of the natural higher bone reformation in the anterior maxilla [18].

A hypothesis to explain these differences could be the substantially different forces acting on the augmentation sites in the timeline of the bone regeneration cycle. While in sinuslifting sites the only recurrent force is the breathing activity creating only linear vertical pressure differences of average 15 mbar with multiple single or multiple peak-pressure changes of 1.5 bar in sneezing activities [33] the subperiosteal augmentation site in the anterior maxilla is exposed to possibly much higher and omnidirectional forces by speaking, smiling, and food-intake activities. This possibly leads to micromotions in the periosteum BiSHB and MoSHB graft interface and in the $\mathrm{BiSHB}$ and MoSHB bone interface that exceed the biological threshold [27-29] and lead to a reduced angiogenesis [35]. This plausible assumption could lead to the observed lesser (bio)mechanical stability of the augmented maxillary bone in the anterior maxilla and might also explain why clinical trials with loose granule biomaterials (xenogeneic and synthetic of various brands) inserted into subperiosteal scaffolds in the anterior maxilla following the procedures described in the literature only for the mandible [19-24] failed from the beginning, led to mere fibrous encapsulation as with nonprimary stable loaded dental implants [29], and had to be canceled. On the other hand, once advanced Platelet Rich Fibrin (aPRF) is added to the MoSHB graft the (bio)mechanical stability of the anterior PeSPTT site slightly supersedes the ITVs obtained in the sinus lift study (Figure 18) and by this strongly backs the evidence, in which PRF induces a faster and better angiogenesis and bone reformation [36-39] alleviating the effects of PeSPTT surgical site micromotions above the biological threshold.

As experimental [30, 31], clinical [33], and the presented studies furthermore suggest, biomaterials containing hydroxyapatite (HA) seem to allow a more mechanical stable bone restoration superior to fast resorbing pure bTCP and to native jawbone, which in the clinical routine is highly welcome for later implant insertion (Figure 19). This fact might be attributed to the very slow resorbing structural dense 


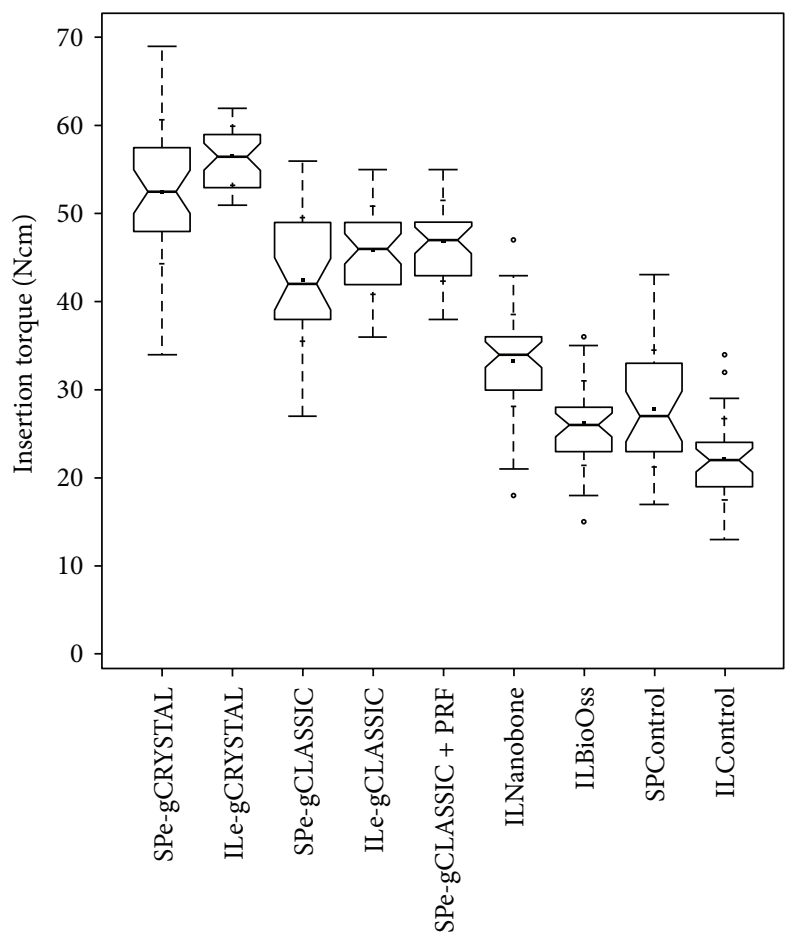

FIGURE 19: Cumulative ITV-comparison notched box plot of the subperiosteal tunnel procedure (SP) in the anterior maxilla and the INTRALIFT-procedure (IL) with BiSHB (e-gCRYSTAL), MoSHB (e-gCLASSIC), MoSHB + Platelet Rich Fibrin (PRF) (e-gCLASSIC + PRF), synthetic granulate $\mathrm{HA} / \mathrm{SiO}_{2}$ (ILNanobone), bovine graft (ILBioOss), control group SP (SPControl), and control group IL (ILControl) depicts the interquartile range (IQR) between the 25 th and 75 th percentile of the specific biomaterial tested where $50 \%$ of the data points were located. Additionally, the upper whiskers represent data within the 75 th percentile +1.5 times the IQR. The lower whisker delimits data of the 25 th percentile -1.5 times the IQR. Circles $\left({ }^{\circ}\right)$ represent the outliers. Within the boxes the notches mark the confidence interval based on the median \pm 1.58 (IQR/sqrt of $n)$ ( $n=$ number of measurements). Additionally, the mean value is indicated by a black square and the cross symbol $(+)$ displays the standard deviation. SPe-gCRYSTAL $(n=36)$, ILe-gCRYSTAL $(n=38)$, SPe-gCLASSIC $(n=35)$, ILe-gCLASSIC $(n=41)$, SPe-grCLASSIC + PRF $(n=38)$, ILNanobone $(n=42)$, ILBioOss $(n=34)$, SPControl $(n=30)$, and ILControl $(n=35)$.

biochemical nature of $\mathrm{HA}$ acting as structural enforcement of the surrounding restored native bone once fully osseointegrated comparable to the tubular HA architecture of the mammal long bones (the tubular HA architecture of long bones (osteon) represents a higher evolutionary stage than the woven bones of the skull and ribs). For the clinician an additive use of BiSHB grafts in conjunction with aPRF for both PeSPTT and tHUCSL-INTRALIFT seems to be preferable in order to achieve the highest possible biomechanical stability of the restored alveolar crest and is currently under clinical investigation by the authors as an extension of the presented study.

\section{Conclusions}

Following the present knowledge of the structure, biomechanics, and physiology of bone regeneration and interpreting the results of the current and prior clinical studies [33], reproducible high clinical success rates in guided bone regeneration and implantology with least patient morbidity depend on several decisive factors in the clinical routine with distinct clinical recommendations and conclusions in respect to the knowledge of the biology of bone healing, regeneration, and restoration.
5.1. Atraumatic and Precise Surgery on Macroscopic and Microscopic Level. Since interruption of vascularization might lead to insufficient angiogenesis in the mostly vast augmentation sites counting up to necessary bone volumes of $2 \mathrm{ccm}$ which are beyond "critical size defects" [34], as least blood vessels as possible should be interrupted by avoidance of fullthickness mucoperiosteal flaps in favor of subperiosteal tunnel or pocket techniques.

The peri- and endosteum-connecting Sharpey fibers should be cleanly cut instead of ripped and torn preferably with the aid of ultrasonic surgical tools ("Piezotomes") that utilize the cavitation effect for a clean periosteal detachment macroscopically (Figures 3 and 4) and microscopically (Figure 1) [7], leaving the cambium layer ("cradle of osteoblasts") undisrupted and fully intact on the cellular and functional level. Piezotome surgery is now widely suggested to generally preserve soft tissues and avoid mechanical damage [49-51], reduce tissue ischemia [52], and stimulate mesenchymal stem cell differentiation and bone healing [53-55].

The consistent results of the present study in company with the results of prior similar studies $[17,33,50,51]$ seem to support the suggestion that utilizing Piezotome surgery for preparation of the augmentation site might be the first step to achieve constant high success rates and does not 
require extensive manual training of the surgeon compared to traditional complex and manually challenging surgical techniques.

5.2. Sufficient Immobilization of the Augmentation Site. Surgical created subperiosteal and endosteal scaffolds for bone augmentation have to be perceived by the clinician more as "fracture sites" similar to "natural" fractures of the jawbones and its mandatory immobilization for proper bone healing. Minimal invasive surgical techniques such as tHUCSLINTRALIFT [56] and the Piezotome-enhanced subperiosteal tunnel or pocket technique (PeSPTT) do not allow the use of autografted, xenogeneic, or synthetic solid bone blocks since they have to be stabilized by osteosynthesis screws. Loose autologous, xenogeneic, or synthetic granulate biomaterials are prone to macro- and micromovements by muscular, breathing (in case of the maxillary sinus), and/or soft tissue forces in the normal function of the stomatognathic and respiratory system and by this might counteract the proper immobilization of the augmentation scaffold as it was suggested by the results presented in a prior study [33]. Therefore the new class of in situ moldable and self-hardening biomaterials used in this study seems to be highly suitable in minimal invasive guided bone regeneration to achieve reproducible good results providing a sufficient and (bio)mechanical highly stable dental implant site of sufficient dimensions. This class of biomaterials seems to fulfill the biological requirement of proper immobilization of the augmentation scaffold for an undisturbed natural bone regeneration below the proposed threshold of micromobility of 100-150 $\mu \mathrm{m}$ [29] in its own final bone-block-like microporous consistence and its immobility on the bone and periosteal surface.

5.3. Use of Biologic Active Autologous Agents Such as Platelet Rich Plasma (PRP) or Advanced Platelet Rich Fibrin (aPRF). It can be considered as a biological fact that all biocompatible materials (i.e., titanium, $\mathrm{ZiO}, \mathrm{PEEK}$, lactic-acid polymers, bioglasses, bTCP, and natural or synthetic hydroxyapatite, etc.) are allowed to osseointegrate but are not "osteoinductive/conductive" per se. Differences between various origins (human, bovine, equine, porcine, all deriving from the same evolutionary stem of mammals, algae, ceramics, glasses, bTCP, and HA) and brands of biomaterials in experimental microscopic, radiologic, clinical, and statistical evaluation on resulting bone regeneration outcome rarely have a significant impact on the work of the clinician since both animal and clinical human studies almost never report if the basal cambium layer of the periosteum was intact and if and how the periosteum was slotted to achieve a tensionless wound closure.

While the postulated coverage of any augmentation site with barrier membranes of different chemical origin (PTFE, titanium, polylactic acid, collagen, etc.) is still favorable in cases of preexisting and/or iatrogenic devastation and destruction of the periosteum, it should be considered as contraindication when the periosteum is detached without lesions from the bone [57].
The better alternative in both cases could be the general replacement of barrier membranes by autologous aPRF membranes that provide a proven physiological enhancement of angiogenesis and bone growth [36-39], copying, and, by concentrating the active biological agents through centrifugation, multiplying the effect of the natural fibrin-condensation on and in every soft- and hard-tissue wound and biocompatible implant materials. Contrary to the production of PRPconcentrates the preparation of aPRF integrates easily in the timely flow of GBR surgeries and does not constitute a challenge to the performing surgeon.

The results of the current study back the experimental findings concerning the advantages for more reliable bone regeneration when $\mathrm{APRF}$ is used and suggest aPRF to enhance biomechanical bone quality to a constant higher level in the clinical routine in conjunction with Piezotome surgery and BiSHB grafts to possibly achieve better and more consistent results with less patient's morbidity compared to traditional methods.

5.4. Paradigm Change of Understanding Guided Bone Regeneration Procedures in the Clinical Practice. Oral surgeons are suggested to turn away from a (bio)material and technical surgical instruments centered view back to a more general view on GBR surgeries based on the biological and physiological facts of bone-healing mechanisms. As it is in diagnostics and surgical planning, plain clinicians rely more and more on prefabricated recipes and software-based surgical "aids" losing out of sight the individual stomatognathic dynamics of the individual patient. Surgical planning and surgery performance should strictly follow the guidelines given by biology and dynamics of the stomatognathic system instead of prefabricated "surgical templates." The suggestion to define autologous bone grafts as "gold-standard" in dental regenerative therapies cannot be backed any more [58] and it should stay a philosophical discussion whether regenerated/ augmented bone should be only native bone or an obviously mechanical more resistive compound of native bone and synthetic biomaterials ("foreign body argument") to sustain the introduced forces by other "foreign bodies," that is, dental implants (made of titanium, $\mathrm{ZiO}, \mathrm{PEEK}$, etc.).

\section{Conflict of Interests}

The authors declare that there is no conflict of interests regarding the publication of this paper.

\section{References}

[1] G. H. Bourne, "The relative importance of periosteum and endosteum in bone healing and the relationship of vitamin C to their activities," Proceedings of the Royal Society of Medicine, vol. 37, no. 6, pp. 275-279, 1944.

[2] D. Özçelik, T. Turan, F. Kabukcuoglu et al., "Bone induction capacity of the periosteum and neonatal dura in the setting of the rat zygomatic arch fracture model," Archives of Facial Plastic Surgery, vol. 5, no. 4, pp. 301-308, 2003.

[3] T. Ortak, R. Özdemir, A. Uysal et al., "Osteogenic capacities of periost grafts, periost flaps and prefabricated periosteal flaps: 
experimental study," Journal of Craniofacial Surgery, vol. 16, no. 4, pp. 594-600, 2005.

[4] C. A. Squier, S. Ghoneim, and C. R. Kremenak, "Ultrastructure of the periosteum from membrane bone," Journal of Anatomy, vol. 171, pp. 233-239, 1990.

[5] S. Srouji, T. Kizhner, D. Ben David, M. Riminucci, P. Bianco, and E. Livne, "The schneiderian membrane contains osteoprogenitor cells: in vivo and in vitro study," Calcified Tissue International, vol. 84, no. 2, pp. 138-145, 2009.

[6] A. Troedhan, A. Kurrek, and M. Wainwright, "Biological principles and physiology of bone regeneration under the schneiderian membrane after sinus lift surgery: a radiological study in 14 patients treated with the transcrestal hydrodynamic ultrasonic cavitational sinus lift (Intralift)," International Journal of Dentistry, vol. 2012, Article ID 576238, 12 pages, 2012.

[7] A. Troedhan, A. Kurrek, M. Wainwright, and S. Jank, "Schneiderian membrane detachment using transcrestal Hydrodynamic Ultrasonic Cavitational Sinus Lift: a human cadaver head study and histologic analysis," Journal of Oral and Maxillofacial Surgery, vol. 72, no. 8, pp. 1503.e1-1503.e10, 2014.

[8] P. J. Boyne, L. C. Lilly, R. E. Marx et al., "De novo bone induction by recombinant human bone morphogenetic protein-2 (rhBMP-2) in maxillary sinus floor augmentation," Journal of Oral and Maxillofacial Surgery, vol. 63, no. 12, pp. 1693-1707, 2005.

[9] D. G. Smiler and R. E. Holmes, "Sinus lift procedure using porous hydroxyapatite: a preliminary clinical report," The Journal of Oral Implantology, vol. 13, no. 2, pp. 239-253, 1986.

[10] M. S. Block and J. N. Kent, "Sinus augmentation for dental implants: the use of autogenous bone," Journal of Oral and Maxillofacial Surgery, vol. 55, no. 11, pp. 1281-1286, 1997.

[11] G. Cordioli, C. Mazzocco, E. Schepers, E. Brugnolo, and Z. Majzoub, "Maxillary sinus floor augmentation using bioactive glass granules and autogenous bone with simultaneous implant placement," Clinical Oral Implants Research, vol. 12, no. 3, pp. 270-278, 2001.

[12] G. V. Cammack II, M. Nevins, D. S. Clem III, J. P. Hatch, and J. T. Mellonig, "Histologic evaluation of mineralized and demineralized freeze-dried bone allograft for ridge and sinus augmentations," International Journal of Periodontics and Restorative Dentistry, vol. 25, no. 3, pp. 231-237, 2005.

[13] S. A. Zijderveld, I. R. Zerbo, J. P. van den Bergh, E. A. Schulten, and C. M. ten Bruggenkate, "Maxillary sinus floor augmentation using a beta-tricalcium phosphate (Cerasorb) alone compared to autogenous bone grafts," The International Journal of Oral \& Maxillofacial Implants, vol. 20, no. 3, pp. 432440, 2005.

[14] G. Szabó, L. Huys, P. Coulthard et al., "A prospective multicenter randomized clinical trial of autogenous bone versus $\beta$ tricalcium phosphate graft alone for bilateral sinus elevation: histologic and histomorphometric evaluation," International Journal of Oral \& Maxillofacial Implants, vol. 20, no. 3, pp. 371381, 2005.

[15] S. S. Wallace and S. J. Froum, "Effect of maxillary sinus augmentation on the survival of endosseous dental implants. A systematic review," Annals of Periodontology, vol. 8, no. 1, pp. 328-343, 2003.

[16] F. Stelzle and K.-U. Benner, "Evaluation of different methods of indirect sinus floor elevation for elevation heights of $10 \mathrm{~mm}$ : an experimental ex vivo study," Clinical Implant Dentistry and Related Research, vol. 13, no. 2, pp. 124-133, 2011.
[17] A. Troedhan, A. Kurrek, M. Wainwright, I. Schlichting, B. Fischak-Treitl, and M. Ladentrog, "The transcrestal hydrodynamic ultrasonic cavitational sinuslift: results of a 2 -year prospective multicentre study on 404 patients, 446 sinuslift sites and 637 inserted implants," Open Journal of Stomatology, vol. 3, no. 9, pp. 471-485, 2013.

[18] S. S. Huja, S. A. Fernandez, K. J. Hill, and Y. Li, "Remodeling dynamics in the alveolar process in skeletally mature dogs," The Anatomical Record Part A: Discoveries in Molecular, Cellular, and Evolutionary Biology, vol. 288, no. 12, pp. 1243-1249, 2006.

[19] J. N. Kent, J. H. Quinn, M. F. Zide, I. M. Finger, M. Jarcho, and S. S. Rothstein, "Correction of alveolar ridge deficiencies with nonresorbable hydroxylapatite," The Journal of the American Dental Association, vol. 105, no. 6, pp. 993-1001, 1982.

[20] S. S. Rothstein, D. A. Paris, and M. P. Zacek, "Use of hydroxylapatite for the augmentation of deficient alveolar ridges," Journal of Oral and Maxillofacial Surgery, vol. 42, no. 4, pp. 224-230, 1984.

[21] R. H. Propper, "A technique for controlled placement of hydroxylapatite over atrophic mandibular ridges," Journal of Oral and Maxillofacial Surgery, vol. 43, no. 6, pp. 469-470, 1985.

[22] F. Härle and T. Kreusch, "Augmentation of the alveolar ridges with hydroxylapatite in a Vicryl tube," International Journal of Oral and Maxillofacial Surgery, vol. 20, no. 3, pp. 144-148, 1991.

[23] Y. Kurihara, T. Wakatsuki, Y. Harada et al., "Mandibular alveolar ridge extension method using a surgical splint with porous hydroxyapatite (HAP) particles," The Bulletin of Tokyo Dental College, vol. 32, no. 2, pp. 71-79, 1991.

[24] M. L. Nevins, M. Camelo, P. Schupbach, B. Friedland, J. M. B. Camelo, and D. M. Kim, "Minimally invasive alveolar ridge augmentation procedure (tunneling technique) using rhPDGFBB in combination with three matrices: a case series," The International Journal of Periodontics \& Restorative Dentistry, vol. 29, no. 4, pp. 371-383, 2009.

[25] M. Nevins, D. Garber, J. J. Hanratty et al., "Human histologic evaluation of anorganic bovine bone mineral combined with recombinant human platelet-derived growth factor BB in maxillary sinus augmentation: case series study," The International Journal of Periodontics \& Restorative Dentistry, vol. 29, no. 6, pp. 583-591, 2009.

[26] E. Martínez-Sanz, O. P. Varghese, M. Kisiel et al., "Minimally invasive mandibular bone augmentation using injectable hydrogels," Journal of Tissue Engineering and Regenerative Medicine, vol. 6, no. 3, pp. s15-s23, 2012.

[27] P. Aspenberg, S. Goodman, S. Toksvig-Larsen, L. Ryd, and T. Albrektsson, "Intermittent micromotion inhibits bone ingrowth. Titanium implants in rabbits," Acta Orthopaedica Scandinavica, vol. 63, no. 2, pp. 141-145, 1992.

[28] M. Jasty, C. Bragdon, D. Burke, D. O'Connor, J. Lowenstein, and W. H. Harris, "In vivo skeletal responses to porous-surfaced implants subjected to small induced motions," The Journal of Bone and Joint Surgery-American Volume, vol. 79, no. 5, pp. 707-714, 1997.

[29] S.-S. Gao, Y.-R. Zhang, Z.-L. Zhu, and H.-Y. Yu, "Micromotions and combined damages at the dental implant/bone interface," International Journal of Oral Science, vol. 4, no. 4, pp. 182-188, 2012.

[30] P. R. Schmidlin, F. Nicholls, A. Kruse, R. A. Zwahlen, and F. E. Weber, "Evaluation of moldable, in situ hardening calcium phosphate bone graft substitutes," Clinical Oral Implants Research, vol. 24, no. 2, pp. 149-157, 2013. 
[31] I. Binderman, A. Yaffe, Y. Samuni, H. Bahar, J. Choukroun, and P. Russe, "Tissue engineering of bone: critical evaluation of scaffold selection," in Bone Regeneration, InTech, 2012.

[32] H. M. Frost, "A 2003 update of bone physiology and Wolff's law for clinicians," The Angle Orthodontist, vol. 74, no. 1, pp. 3-15, 2004.

[33] A. Troedhan, I. Schlichting, A. Kurrek, and M. Wainwright, "Primary implant stability in augmented sinuslift-sites after completed bone regeneration: a randomized controlled clinical study comparing four subantrally inserted biomaterials," Scientific Reports, vol. 4, article 5877, 2014.

[34] A. Udagawa, S. Sato, A. Hasuike, M. Kishida, Y. Arai, and K. Ito, "Micro-CT observation of angiogenesis in bone regeneration," Clinical Oral Implants Research, vol. 24, no. 7, pp. 787-792, 2013.

[35] A. Mammoto, K. M. Connor, T. Mammoto et al., "A mechanosensitive transcriptional mechanism that controls angiogenesis," Nature, vol. 457, no. 7233, pp. 1103-1108, 2009.

[36] T. Mammoto, A. Jiang, E. Jiang, and A. Mammoto, "Platelet rich plasma extract promotes angiogenesis through the angiopoietin1-Tie2 pathway," Microvascular Research, vol. 89, pp. 15-24, 2013.

[37] A. Ballini, M. Scivetti, S. Cantore, B. Rapone, G. Favia, and F. R. Grassi, "Tissuue engineering in maxillar sinus lifting: a comparation of differents grafts and confocal laser scanning microscopic evaluation," in Bone Regeneration, H. Tal, Ed., chapter 5, InTech, Rijeka, Croatia, 2012.

[38] C. L. M. Bao, E. Y. Teo, M. S. Chong, Y. Liu, M. Choolani, and J. K. Chan, "Advances in bone tissue engineering," in Regenerative Medicine and Tissue Engineering, pp. 599-614, InTech, Rijeka, Croatia, 2013.

[39] N. Lohse, J. Schulz, and H. Schliephake, "Effect of fibrin on osteogenic differentiation and VEGF expression of bone marrow stromal cells in mineralised scaffolds: a three-dimensional analysis," European Cells \& Materials, vol. 23, pp. 413-424, 2012.

[40] Z. Wang, Y. Weng, S. Lu et al., "Osteoblastic mesenchymal stem cell sheet combined with Choukroun platelet-rich fibrin induces bone formation at an ectopic site," Journal of Biomedical Materials Research Part B: Applied Biomaterials, 2014.

[41] I. Turkyilmaz and E. A. McGlumphy, "Influence of bone density on implant stability parameters and implant success: a retrospective clinical study," BMC Oral Health, vol. 8, no. 1, article 32, 2008.

[42] P. Trisi, G. Perfetti, E. Baldoni, D. Berardi, M. Colagiovanni, and G. Scogna, "Implant micromotion is related to peak insertion torque and bone density," Clinical Oral Implants Research, vol. 20, no. 5, pp. 467-471, 2009.

[43] L. R. Walker, G. A. Morris, and P. J. Novotny, "Implant insertional torque values predict outcomes," Journal of Oral and Maxillofacial Surgery, vol. 69, no. 5, pp. 1344-1349, 2011.

[44] G. Augustin, A. Antabak, and S. Davila, "The periosteum. Part 1: anatomy, histology and molecular biology," Injury, vol. 38, no. 10, pp. 1115-1130, 2007.

[45] J. E. Aaron, "Periosteal sharpey's fibers: a novel bone matrix regulatory system," Frontiers in Endocrinology, vol. 3, article 98, 2012.

[46] A. C. Vailas, D. M. Deluna, L. L. Lewis, S. L. Curwin, R. R. Roy, and E. K. Alford, "Adaptation of bone and tendon to prolonged hindlimb suspension in rats," Journal of Applied Physiology, vol. 65, no. 1, pp. 373-376, 1988.
[47] T. J. Wronski and E. R. Morey, "Effect of spaceflight on periosteal bone formation in rats," The American Journal of PhysiologyRegulatory Integrative and Comparative Physiology, vol. 244, no. 3, pp. R305-R309, 1983.

[48] T. Matsuura, K. Tokutomi, M. Sasaki, M. Katafuchi, E. Mizumachi, and H. Sato, "Distinct characteristics of mandibular bone collagen relative to long bone collagen: relevance to clinical dentistry," BioMed Research International, vol. 2014, Article ID 769414, 9 pages, 2014.

[49] A. Troedhan, A. Kurrek, and M. Wainwright, "Ultrasonic piezotome surgery: is it a benefit for our patients and does it extend surgery time? A retrospective comparative study on the removal of 100 impacted mandibular 3rd molars," Open Journal of Stomatology, vol. 1, no. 4, pp. 179-184, 2011.

[50] M. Goyal, K. Marya, A. Jhamb et al., "Comparative evaluation of surgical outcome after removal of impacted mandibular third molars using a Piezotome or a conventional handpiece: a prospective study," British Journal of Oral and Maxillofacial Surgery, vol. 50, no. 6, pp. 556-561, 2012.

[51] S. Pappalardo and R. Guarnieri, "Randomized clinical study comparing piezosurgery and conventional rotatory surgery in mandibular cyst enucleation," Journal of Cranio-Maxillofacial Surgery, vol. 42, no. 5, pp. e80-e85, 2013.

[52] V. N. Suchkova, R. B. Baggs, S. K. Sahni, and C. W. Francis, "Ultrasound improves tissue perfusion in ischemic tissue through a nitric oxide dependent mechanism," Thrombosis and Haemostasis, vol. 88, no. 5, pp. 865-870, 2002.

[53] N. Doan, P. Reher, S. Meghji, and M. Harris, "In vitro effects of therapeutic ultrasound on cell proliferation, protein synthesis, and cytokine production by human fibroblasts, osteoblasts, and monocytes," Journal of Oral and Maxillofacial Surgery, vol. 57, no. 4, pp. 409-420, 1999.

[54] S. Mouraret, K. S. Houschyar, D. J. Hunter et al., "Cell viability after osteotomy and bone harvesting: comparison of piezoelectric surgery and conventional bur," International Journal of Oral \& Maxillofacial Surgery, vol. 43, no. 8, pp. 966-971, 2014.

[55] J. Reside, E. Everett, R. Padilla et al., "In vivo assessment of bone healing following piezotome ultrasonic instrumentation," Clinical Implant Dentistry and Related Research, 2013.

[56] A. C. Troedhan, A. Kurrek, M. Wainwright, and S. Jank, "Hydrodynamic ultrasonic sinus floor elevation-an experimental study in sheep," Journal of Oral and Maxillofacial Surgery, vol. 68, no. 5, pp. 1125-1130, 2010.

[57] E. A. J. M. Schulten, H.-J. Prins, J. R. Overman, M. N. Helder, C. M. ten Bruggenkate, and J. Klein-Nulend, "A novel approach revealing the effect of a collagenous membrane on osteoconduction in maxillary sinus floor elevation with $\beta$ tricalcium phosphate," European Cells and Materials, vol. 25, pp. 215-228, 2012.

[58] M. del Fabbro, G. Rosano, and S. Taschieri, "Implant survival rates after maxillary sinus augmentation," European Journal of Oral Sciences, vol. 116, no. 6, pp. 497-506, 2008. 

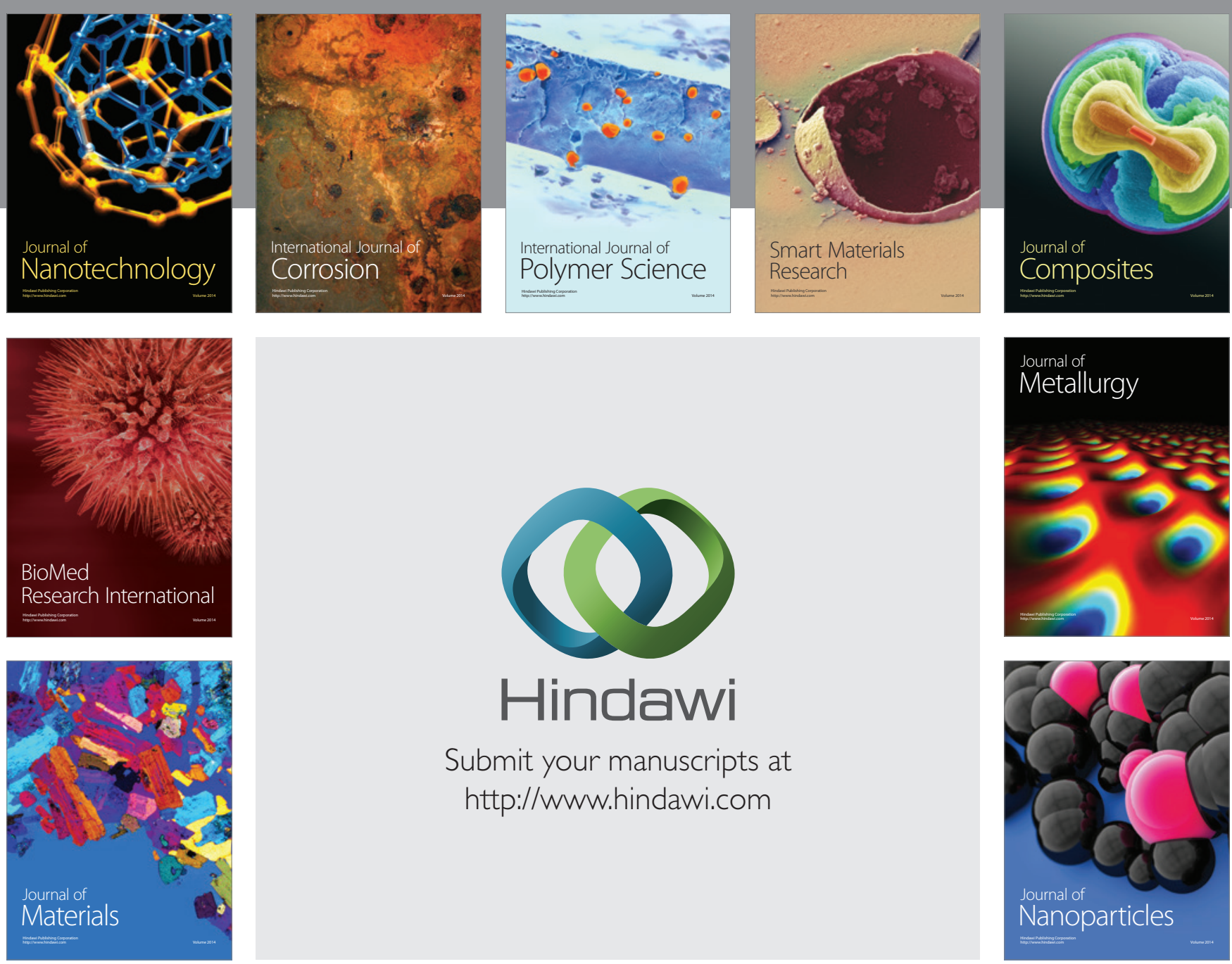

Submit your manuscripts at http://www.hindawi.com
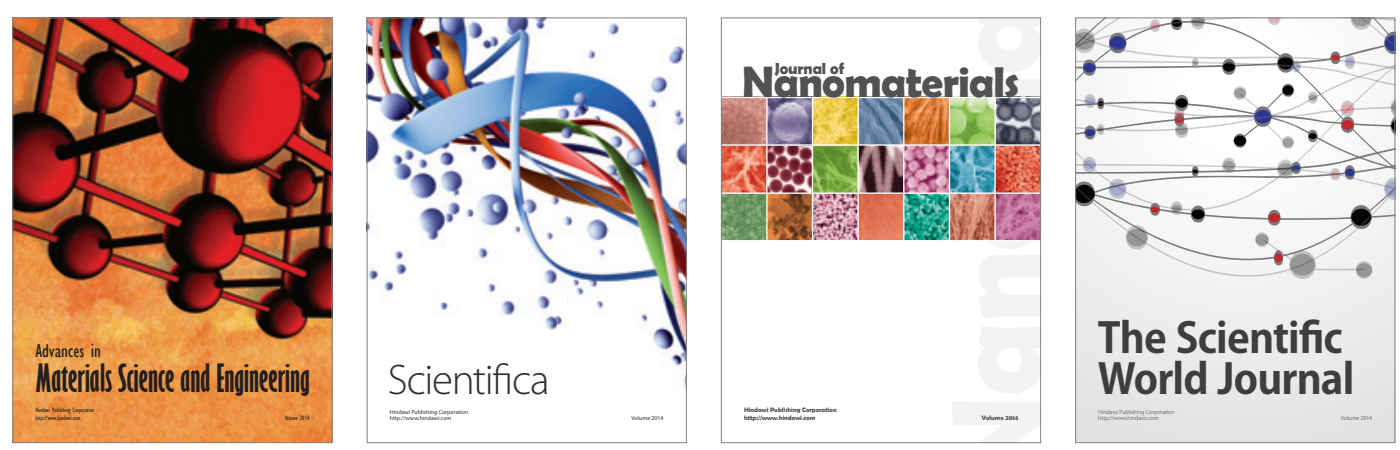

\section{The Scientific World Journal}
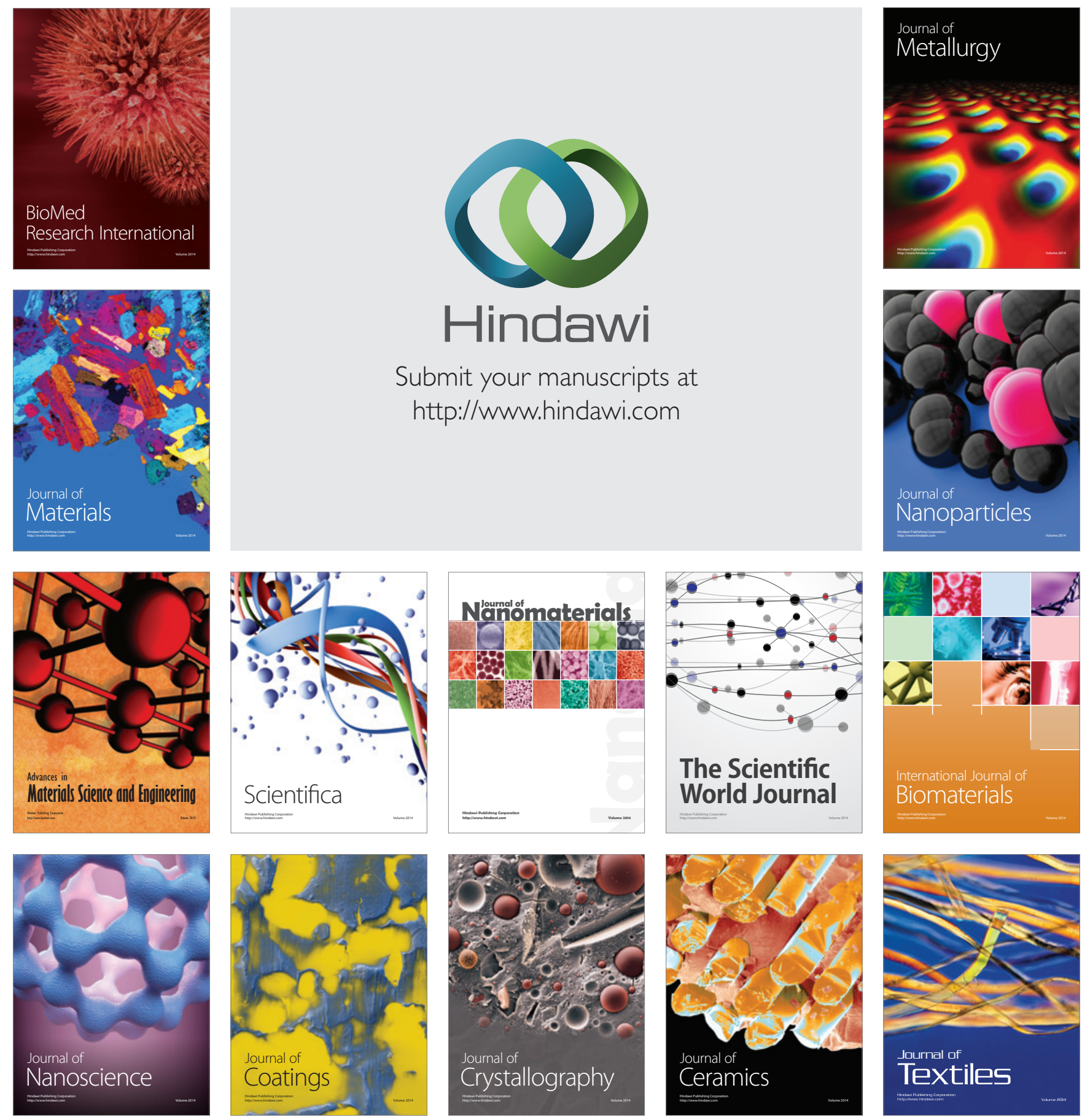